\title{
GUARANTEED AND ROBUST A POSTERIORI ERROR ESTIMATES AND BALANCING DISCRETIZATION AND LINEARIZATION ERRORS FOR MONOTONE NONLINEAR PROBLEMS
}

\author{
LINDA EL ALAOUI ${ }^{1}$, ALEXANDRE ERN $^{2}$, AND MARTIN VOHRALÍK ${ }^{3}$
}

${ }^{1}$ LAGA, Université Paris 13, 99 avenue Jean-Baptiste Clément, 93430 Villetaneuse, France e-mail: elalaoui@math.univ-paris13.fr

${ }^{2}$ Université Paris-Est, CERMICS, Ecole des Ponts, 6 \& 8 av. B. Pascal, 77455 Marne-la-Vallée, France e-mail: ern@cermics.enpc.fr

${ }^{3}$ UPMC Univ. Paris 06, UMR 7598, Laboratoire Jacques-Louis Lions, 75005, Paris, France \&

CNRS, UMR 7598, Laboratoire Jacques-Louis Lions, 75005, Paris, France e-mail: vohralik@ann.jussieu.fr

\begin{abstract}
We derive a posteriori error estimates for a class of second-order monotone quasi-linear diffusion-type problems approximated by piecewise affine, continuous finite elements. Our estimates yield a guaranteed and fully computable upper bound on the error measured by the dual norm of the residual, as well as a global error lower bound, up to a generic constant independent of the nonlinear operator. They are thus fully robust with respect to the nonlinearity, thanks to the choice of the error measure. They are also locally efficient, albeit in a different norm, and hence suitable for adaptive mesh refinement. Moreover, they allow to distinguish, estimate separately, and compare the discretization and linearization errors. Hence, the iterative (Newton-Raphson, quasi-Newton) linearization can be stopped whenever the linearization error drops to the level at which it does not affect significantly the overall error. This can lead to important computational savings, as performing an excessive number of unnecessary linearization iterations can be avoided. Numerical experiments for the $p$-Laplacian illustrate the theoretical developments.
\end{abstract}

Key words: a posteriori error estimate, monotone nonlinear problem, linearization, balancing errors, guaranteed upper bound, robustness

\section{INTRODUCTION}

Let $\Omega$ be an open polyhedron of $\mathbb{R}^{d}, d \geq 2$. We consider the nonlinear problem in conservative form

$$
\begin{aligned}
& -\nabla \cdot \boldsymbol{\sigma}(\nabla u)=f \quad \text { in } \Omega, \\
& u=0 \quad \text { on } \partial \Omega \text {. }
\end{aligned}
$$

The scalar-valued unknown function $u$ is termed the potential, and the $\mathbb{R}^{d}$-valued function $-\sigma(\nabla u)$ is termed the flux. We assume that the flux function $\sigma: \mathbb{R}^{d} \rightarrow \mathbb{R}^{d}$ takes the following quasi-linear form

$$
\forall \boldsymbol{\xi} \in \mathbb{R}^{d}, \quad \boldsymbol{\sigma}(\boldsymbol{\xi})=a(|\boldsymbol{\xi}|) \boldsymbol{\xi},
$$

where $|\cdot|$ denotes the Euclidean norm in $\mathbb{R}^{d}$ and where $a: \mathbb{R}_{+} \rightarrow \mathbb{R}$ is a given function. The function $a$ is assumed below to satisfy a growth condition of the form $a(x) \sim x^{p-2}$ as $x \rightarrow+\infty$ for some real

THIS WORK WAS PARTIALLY SUPPORTED BY THE GNR MOMAS (PACEN/CNRS, ANDRA, BRGM, CEA, EDF, IRSN, FRANCE) 
number $p \in(1,+\infty)$, so that the natural energy space $V$ for the above model problem is the Sobolev space $W_{0}^{1, p}(\Omega)$. The data $f$ is taken in $L^{q}(\Omega)$ where $q:=\frac{p}{p-1}$ so that $\frac{1}{p}+\frac{1}{q}=1$. Hence, the model problem in weak form amounts to finding $u \in V$ such that

$$
(\boldsymbol{\sigma}(\nabla u), \nabla v)=(f, v) \quad \forall v \in V
$$

where $(\cdot, \cdot)$ denotes the integral over $\Omega$ of the (scalar) product of the two arguments. The function $a$ satisfies monotonicity and continuity conditions stated in Section 2 below and ensuring that the problem (1.3) is well-posed.

The prototypical example for the present model problem is the so-called $p$-Laplacian, for which $a(x)=$ $x^{p-2}$. The a priori error analysis for approximating the $p$-Laplacian by piecewise affine, continuous finite elements has been started by Glowinski and Marrocco [19, 20]; see also Ciarlet [13, p. 312]. One wellknown difficulty when working with the natural energy norm is that the derived error estimates are not sharp. This drawback has been circumvented by Barrett and Liu [6] upon introducing a so-called quasinorm, thereby achieving optimal approximation results. The quasi-norm of the error between the exact solution $u$ and the approximation solution, say $u_{h}$, is a weighted $L^{2}$-norm of the gradient $\nabla\left(u-u_{h}\right)$, where the weight depends on $\nabla u$ and $\nabla u_{h}$.

The a posteriori error analysis of finite element approximations to a large class of nonlinear problems, including the present model problems, has been started by Verfürth; see [29] and [30, p. 47]. The main result is a two-sided bound of the energy error by the dual norm of the residual multiplied by suitable norms of the linearized operator at the exact solution, under the assumption that this latter operator is invertible and locally Lipschitz-continuous and that the approximate solution is sufficiently close to the exact solution. This yields in particular residual-based estimators in the energy norm. These estimators have been exploited, in particular, by Veeser [28] to prove the convergence of an adaptive finite element method for the $p$-Laplacian. Alternatively, quasi-norm error estimates for the $p$-Laplacian have been analyzed by Liu and Yan [24, 25, 26], leading to weighted residual-based estimators. Quasi-norm residualbased estimators have been further explored by Carstensen and Klose [9] with a focus on evaluating the constants in the estimates and under the assumption that the gradient norm of the approximate solution is positive everywhere in the domain. Moreover, gradient recovery techniques have been analyzed by Carstensen, Liu, and Yan [10] to estimate the quasi-norm of the error. Quite recently, Diening and Kreuzer [16] have obtained two-sided bounds for an appropriate measure of the error and proven the linear convergence of a suitable adaptive finite element method. The error measure is the $L^{2}$-norm of the difference $\mathbf{F}(\nabla u)-\mathbf{F}\left(\nabla u_{h}\right)$, where the auxiliary vector field $\mathbf{F}$ is such that $\mathbf{F}(\boldsymbol{\xi})=|\boldsymbol{\sigma}(\boldsymbol{\xi})|^{1 / 2}|\boldsymbol{\xi}|^{-1 / 2} \boldsymbol{\xi}$. This error measure turns out to be equivalent to the quasi-norm of the error, with constants depending on the nonlinearity (that is, the properties of the function $a$ in (1.2)).

We observe that, whatever the error measure, the above bounds on the error involve constants depending on the function $a$. In the case of the $p$-Laplacian, they depend on the Lebesgue exponent $p$. Moreover, with a few exceptions, e.g., [9, 10], the error upper bounds involve generic constants, making them unsuitable for actual error control. Therefore, the first objective of this work is to derive guaranteed and robust bounds on the error, that is, error upper bounds without undetermined constants and twosided error bounds independent of the nonlinearity. To this purpose, we use as error measure a residual flux-based dual norm, namely

$$
J_{u}\left(u_{h}\right):=\left\|\nabla \cdot \boldsymbol{\sigma}(\nabla u)-\nabla \cdot \boldsymbol{\sigma}\left(\nabla u_{h}\right)\right\|_{V^{\prime}}=\sup _{v \in V \backslash\{0\}} \frac{\left(\boldsymbol{\sigma}(\nabla u)-\boldsymbol{\sigma}\left(\nabla u_{h}\right), \nabla v\right)}{\|v\|_{V}} .
$$

Working with residual flux-based quantities to measure the error is somewhat natural since fluxes satisfy basic conservation properties that are at the heart of the design of approximation methods, even using continuous finite elements. Furthermore, the idea of using a dual norm is inspired by the work of Verfürth where dual norms have been considered, e.g., in the context of parabolic [34] and convectiondominated stationary convection-diffusion equations [36]. Dual residual norms have also been considered for nonlinear problems in [29], and the present dual norm has been considered in [11, 12]. More recently, 
it has been observed in [40] that residual-flux based error measures are also natural in the context of diffusion problems with heterogeneous coefficients. Furthermore, we remark that although our error upper bounds are fully computable, the actual error measure is not, even if the exact solution is known; we will discuss below how the error measure can be approximated in numerical experiments with synthetic exact solutions so as to compute effectivity indices. Note, however, that in practical computations, the exact solution is never known and hence the error is never computable. We also point out that achieving robust error estimates does not mean necessarily that the error bounds can be extended to the limit cases $p=1$ or $p=+\infty$, similarly to the vanishing-diffusion limit in convection-diffusion equations, since the approximation method may fail to converge. Our a posteriori error estimates are formulated in terms of a conforming flux reconstruction, say $\mathbf{t}_{h}$, similarly to earlier work in the linear $[1,7,14,23,27,40]$ and nonlinear [22] cases. The error upper bound hinges on a local conservation property of the reconstructed flux $\mathbf{t}_{h}$; see Assumption 3.4 below. The error lower bound hinges instead on an approximation property of $\mathbf{t}_{h}$; see Assumption 4.1 below. In the present work, we provide two examples for reconstructing the flux $\mathbf{t}_{h}$ in the context of piecewise affine, continuous finite elements satisfying Assumptions 3.4 and 4.1. Higher-order methods are not considered herein. This is motivated, in part, by the fact that in many cases the exact solution $u$ may not have much additional regularity beyond that of the natural energy space $V$; see [13, p. 324] for a similar remark concerning the $p$-Laplacian.

The discrete problem amounts to a system of nonlinear equations, and, in practice, is solved using an iterative method involving some kind of linearization. Given an approximate solution, say $u_{L, h}$, at a given stage of the iterative process and on a given mesh, there are actually two sources of error, namely linearization and discretization. Balancing these two sources of error can be of paramount importance in practice, since it can avoid performing an excessive number of nonlinear solver iterations if the discretization error dominates. Therefore, the second objective of this work is to design a posteriori error estimates distinguishing linearization and discretization errors in the context of an adaptive procedure. This type of analysis has been started by Chaillou and Suri $[11,12]$ for a certain class of nonlinear problems similar to the present one and in the context of iterative solution of linear algebraic systems in [21]. Chaillou and Suri only considered a fixed stage of the linearization process, while we take here the analysis one step further in the context of an iterative loop. Furthermore, they only considered a specific form for the linearization, namely of quasi-Newton type, while we allow for a wider choice, including NewtonRaphson methods. We consider an adaptive loop in which at each step, a fixed mesh is considered and the nonlinear solver is iterated until the linearization error estimate is brought below the discretization error estimate; then, the mesh is adaptively refined and the loop is advanced. In this work, we will not tackle the delicate issue of proving the convergence of the above adaptive algorithm. We will also assume that at each iterate of the nonlinear solver, a well-posed problem is obtained. This property is by no means granted in general; it amounts, for the $p$-Laplacian, to assume, as mentioned before in [9], that the gradient norm of the approximate solution is positive everywhere in the domain. We mention that in our numerical experiments, all the discrete problems were indeed found to be well-posed.

This paper is organized as follows. Section 2 describes the setting for the nonlinear problem together with its discretization and linearization. Section 3 is devoted to the derivation of the guaranteed error upper bounds, while Section 4 is concerned with the efficiency of the estimators. Section 5 presents two possible approaches to reconstruct the flux $\mathbf{t}_{h}$ in the context of piecewise affine, continuous finite elements. Section 6 contains the numerical results. Finally, Appendix A collects various auxiliary lemmas.

\section{The SETTiNG}

We describe here the considered nonlinear problem together with its discretization and linearization.

2.1. The continuous problem. Henceforth, for a real number $r \in(1,+\infty)$ and a subset $\omega \subset \Omega,\|\cdot\|_{r, \omega}$ denotes the canonical norm in $L^{r}(\omega)$ or $\left[L^{r}(\omega)\right]^{d}$ with appropriate Lebesgue measure; the subscript $\omega$ is omitted whenever $\omega=\Omega$ while the subscript $r$ is omitted whenever $r=2$. Moreover, the space $V$ is 
equipped with the norm $\|\cdot\|_{V}:=\|\nabla(\cdot)\|_{p}$. It is convenient to introduce the operator $A: V \rightarrow V^{\prime}$ such that for all $u, v \in V$,

$$
\langle A u, v\rangle_{V^{\prime}, V}:=(\boldsymbol{\sigma}(\nabla u), \nabla v) .
$$

The function $a$ in the definition (1.2) of the flux function $\sigma$ is assumed to satisfy the following growth condition: There are constants $R_{1}, c_{1}$, and $c_{2}$ such that for all $x \geq R_{1}$,

$$
c_{1} x^{p-2} \leq a(x) \leq c_{2} x^{p-2} .
$$

Owing to the upper bound in (2.2) and using the Hölder inequality, it is readily seen that for all $u \in V$, $\boldsymbol{\sigma}(\nabla u) \in\left[L^{q}(\Omega)\right]^{d}$ so that $A u$ is indeed in $V^{\prime}$. The lower bound in (2.2) is classically used to infer an a priori estimate on the solution to (1.3).

Concerning the operator $A$, we make the following assumptions:

i) Monotonicity. For all $v, w \in V$, there holds

$$
\langle A v-A w, v-w\rangle_{V^{\prime}, V} \geq 0 .
$$

Moreover, there exist a function $\gamma: \mathbb{R}_{+} \rightarrow \mathbb{R}_{+}^{*}$ (taking positive values) and a strictly increasing function $\chi: \mathbb{R}_{+} \rightarrow \mathbb{R}_{+}$such that $\chi(0)=0$ and $\lim _{t \rightarrow+\infty} \chi(t)=+\infty$ such that for all $R \geq 0$ and for all $v, w \in B_{V}(0, R)$, the open ball in $V$ centered at the origin and of radius $R$, there holds

$$
\langle A v-A w, v-w\rangle_{V^{\prime}, V} \geq \gamma(R) \chi\left(\|v-w\|_{V}\right)\|v-w\|_{V} .
$$

ii) Hölder continuity. There exist a function $\Gamma: \mathbb{R}_{+} \rightarrow \mathbb{R}_{+}$and a real number $\alpha \in(0,1]$ such that for all $R \geq 0$ and for all $v, w \in B_{V}(0, R)$,

$$
\|A v-A w\|_{V^{\prime}} \leq \Gamma(R)\|v-w\|_{V}^{\alpha} .
$$

Under these assumptions, the model problem (1.3) is well-posed; see, e.g., [13, p. 321].

Remark 2.1 (Link with a minimization problem). Introducing the energy density $\varphi(x):=\int_{0}^{x} y a(y) \mathrm{d} y$ for $x \in \mathbb{R}_{+}$and the functional

$$
J: V \ni v \longmapsto J(v):=(\varphi(|\nabla v|), 1)-(f, v),
$$

it is readily seen that $J$ is differentiable in $V$ with $J^{\prime}(v)=A v-f$ so that $J$ is convex owing to (2.3). Hence, solving (1.3) amounts to seeking the unique minimizer of the functional $J$ over $V$.

Example 2.2 (p-Laplacian). In the case of the $p$-Laplacian, for $p \geq 2$, the operator $A$ is strongly monotone over the whole space $V$ with $\chi(t)=t^{p-1}$ (the function $\gamma$ in (2.4) is then taken to be constant), while it is Lipschitz-continuous for bounded arguments, that is $\alpha=1$ in $(2.5)$ with $\Gamma(R) \sim(2 R)^{p-2}$. For $p \leq 2$, the operator $A$ is strongly monotone for bounded arguments with $\chi(t)=t$ and $\gamma(R) \sim(2 R)^{p-2}$, while it is Hölder-continuous with parameter $\alpha=p-1$ over the whole space $V$. Furthermore, we mention that the setting for the p-Laplacian fits the more general framework of the so-called $\varphi$-Laplacian; see, e.g., [15].

2.2. Discretization. Let $\left(\mathcal{T}_{h}\right)_{h}$ be a shape-regular family of affine meshes of $\Omega$ consisting of simplices. We assume that the meshes cover $\Omega$ exactly. We also suppose that each mesh $\mathcal{T}_{h}$ is matching in the sense that it contains no "hanging nodes". Let $\mathbb{P}_{k}\left(\mathcal{T}_{h}\right), k \geq 0$, be spanned by piecewise polynomials of total degree $\leq k$ on the mesh $\mathcal{T}_{h}$ and let

$$
V_{h}:=\mathbb{P}_{1}\left(\mathcal{T}_{h}\right) \cap V,
$$

be the usual first-order, continuous finite element space on the mesh $\mathcal{T}_{h}$. The discrete nonlinear problem takes the following form

$$
u_{h} \in V_{h}, \quad\left(\boldsymbol{\sigma}\left(\nabla u_{h}\right), \nabla v_{h}\right)=\left(f, v_{h}\right) \quad \forall v_{h} \in V_{h} .
$$



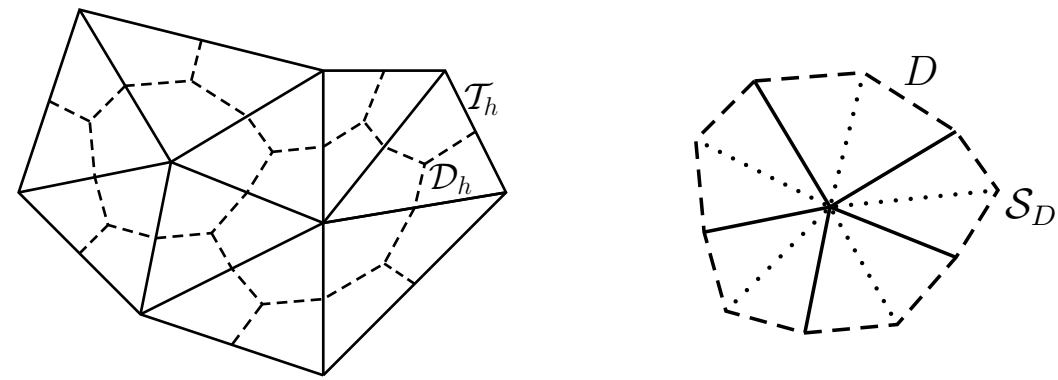

Figure 1. Simplicial mesh $\mathcal{T}_{h}$ and the associated vertex-centered dual mesh $\mathcal{D}_{h}$ (left) and the fine simplicial mesh $\mathcal{S}_{D}$ of $D \in \mathcal{D}_{h}$ (right)

The discrete nonlinear problem (2.8) is well-posed and its solution $u_{h}$ satisfies an a priori estimate similar to that satisfied by the exact solution $u$. The properties of the operator $A$ also imply that $u_{h}$ converges to $u$ in $V$.

In addition to the partition of $\Omega$ induced by the mesh $\mathcal{T}_{h}$, we will consider two other partitions. Firstly, let $\mathcal{D}_{h}$ be the dual mesh formed around the vertices of $\mathcal{T}_{h}$ using element and face barycenters; see the left part of Figure 1 for an illustration in dimension $d=2$. The set $\mathcal{D}_{h}$ is partitioned into $\mathcal{D}_{h}=\mathcal{D}_{h}^{\text {int }} \cup \mathcal{D}_{h}^{\text {ext }}$, where $\mathcal{D}_{h}^{\text {int }}$ collects the dual volumes associated with interior vertices and $\mathcal{D}_{h}^{\text {ext }}$ those associated with boundary vertices. For $D \in \mathcal{D}_{h}, h_{D}$ denotes its diameter. Secondly, $\mathcal{S}_{h}$ is the simplicial mesh which is simultaneously a submesh (refinement) of both $\mathcal{T}_{h}$ and $\mathcal{D}_{h}$; see the right part of Figure 1 . The meshes $\left(\mathcal{S}_{h}\right)_{h}$ are assumed to be shape-regular with parameter denoted by $\kappa$. The mesh $\mathcal{D}_{h}$ will be used in the error upper bound; the error estimators will also be localized on that mesh. The mesh $\mathcal{S}_{h}$ will be used in the construction of the equilibrated flux $\mathbf{t}_{h}$ and in the error lower bound.

The faces of the mesh $\mathcal{S}_{h}$ are collected into the set $\mathcal{G}_{h}$ which is partitioned into $\mathcal{G}_{h}=\mathcal{G}_{h}^{\text {int }} \cup \mathcal{G}_{h}^{\text {ext }}$, where $\mathcal{G}_{h}^{\text {int }}$ collects the faces inside $\Omega$ and $\mathcal{G}_{h}^{\text {ext }}$ those located on the boundary $\partial \Omega$. The set $\mathcal{G}_{h}^{\text {int }}$ is further partitioned into $\mathcal{G}_{h}^{\text {int }}=\mathcal{G}_{h}^{\mathcal{D}} \cup \mathcal{G}_{h}^{\mathcal{T}} \cup \mathcal{G}_{h}^{\mathcal{D} \mathcal{T}}$, where

$$
\begin{aligned}
& \mathcal{G}_{h}^{\mathcal{D}}=\left\{F \in \mathcal{G}_{h}^{\text {int }}, F \subset \partial D \text { for some } D \in \mathcal{D}_{h}\right\}, \\
& \mathcal{G}_{h}^{\mathcal{T}}=\left\{F \in \mathcal{G}_{h}^{\text {int }}, F \subset \partial T \text { for some } T \in \mathcal{T}_{h}\right\} .
\end{aligned}
$$

The set $\mathcal{G}_{h}^{\mathcal{D}}$ collects the interior faces located on the boundary of the dual volumes (indicated by a dashed line in the right part of Figure 1), $\mathcal{G}_{h}^{\mathcal{T}}$ those located on the boundary of the original elements (indicated by a solid line), and $\mathcal{G}_{h}^{\mathcal{D T}}$ the remaining ones (indicated by a dotted line).

2.3. Linearization. The continuous nonlinear problem (1.3) can be linearized at a given function $u_{0} \in V$. To this purpose, a linear or affine flux function $\boldsymbol{\sigma}_{L}: \mathbb{R}^{d} \rightarrow \mathbb{R}^{d}$ is introduced (the dependence of $\boldsymbol{\sigma}_{L}$ on $u_{0}$ is left implicit to alleviate the notation), leading to the continuous linearized problem

$$
u_{L} \in V, \quad\left(\sigma_{L}\left(\nabla u_{L}\right), \nabla v\right)=(f, v) \quad \forall v \in V .
$$

Similarly, for the discrete nonlinear problem (2.8), the discrete linearized problem is

\begin{tabular}{|c|c|c|}
\hline continuous nonlinear (1.3) & discretization & discrete nonlinear (2.8) \\
\hline linearization & \multirow{2}{*}{ discretization } & linearization \\
\hline continuous linearized (2.11) & & discrete linearized (2.12) \\
\hline
\end{tabular}

$$
u_{L, h} \in V_{h}, \quad\left(\sigma_{L}\left(\nabla u_{L, h}\right), \nabla v_{h}\right)=\left(f, v_{h}\right) \quad \forall v_{h} \in V_{h} .
$$

An important property is that this yields the following commuting diagram 
It is convenient to introduce the linearized operator $A_{L}: V \rightarrow V^{\prime}$ such that for all $u, v \in V$,

$$
\left\langle A_{L} u, v\right\rangle_{V^{\prime}, V}:=\left(\sigma_{L}(\nabla u), \nabla v\right) .
$$

In practice, starting from an initial guess $u_{L, h}^{0} \in V_{h}$, a sequence of discrete solutions $\left\{u_{L, h}^{i}\right\}_{i \geq 1}$ is generated through the following iterative algorithm: For $i \geq 1$,

(1) Linearize the flux function at $u_{L, h}^{i-1}$;

(2) Solve the discrete linearized problem (2.12) for $u_{L, h}^{i}$;

(3) If desired precision is reached, then stop, else set $i \leftarrow(i+1)$ and go to step (1).

One of the central points of the present paper is the choice of the stopping criterion. We propose it in Section 3.3 below with the purpose to balance discretization and linearization errors.

There are two basic choices for linearizing the flux function $\sigma_{L}$ at a given $u_{0}$. The first one is,

$$
\forall \boldsymbol{\xi} \in \mathbb{R}^{d}, \quad \boldsymbol{\sigma}_{L}(\boldsymbol{\xi}):=a\left(\left|\nabla u_{0}\right|\right) \boldsymbol{\xi} .
$$

This choice has been considered by Chaillou and Suri [11, 12]. The second one, assuming that $a$ is differentiable on $\mathbb{R}_{+}$and that $x a^{\prime}(x)$ is bounded by $x^{p-2}$ for large $x$, is

$$
\forall \boldsymbol{\xi} \in \mathbb{R}^{d}, \quad \boldsymbol{\sigma}_{L}(\boldsymbol{\xi}):=a\left(\left|\nabla u_{0}\right|\right) \boldsymbol{\xi}+a^{\prime}\left(\left|\nabla u_{0}\right|\right) \frac{1}{\left|\nabla u_{0}\right|}\left(\nabla u_{0} \otimes \nabla u_{0}\right)\left(\boldsymbol{\xi}-\nabla u_{0}\right) .
$$

In the context of the above iterative loop, the choice (2.14) corresponds to a quasi-Newton iteration for solving the discrete nonlinear problem (2.8), while (2.15) corresponds to the full Newton-Raphson iteration. In the case (2.15), we slightly abuse the terminology since the operator $A_{L}$ is actually affine.

Proving the well-posedness of the linearized problems (2.11) and (2.12) and the convergence of the above iterative loop goes beyond the scope of the present paper. Henceforth, we make the assumption that these properties indeed hold. Incidentally, we observe that if the iterative loop converges, then necessarily $u_{L, h}^{i} \rightarrow u_{h}$, the unique solution to (2.8), as $i \rightarrow+\infty$. We also remark that in the context of the $p$-Laplacian, we need to assume that for all $i \geq 0$, the gradient norm of $u_{L, h}^{i}$ is positive everywhere in the domain. In our numerical experiments, all the discrete problems were indeed found to be well-posed, and the iterative loop did converge. Actually, the choice (2.15) associated with the Newton-Raphson iteration led to much faster convergence rates than the choice (2.14) associated with the quasi-Newton iteration.

Remark 2.3 (Anisotropic diffusion for Newton-Raphson linearization). We observe that the choice (2.15) for the linearized flux function amounts to adding anisotropic (rank-one) diffusion to the isotropic diffusion $a\left(\left|\nabla u_{0}\right|\right)$ obtained using the linearization (2.14).

\section{A POSTERIORI ERROR ESTIMATES}

We state and prove here our a posteriori error estimates and give our stopping criterion for iterative linearizations.

Recalling the definition of the error measure (1.4) and that of the energy norm, and using the definition (2.1) of the nonlinear operator $A$, the goal of this section is to derive guaranteed upper bounds for the quantity

$$
\mathcal{J}_{u}\left(u_{L, h}\right):=\left\|A u-A u_{L, h}\right\|_{V^{\prime}}=\sup _{v \in V \backslash\{0\}} \frac{\left(\boldsymbol{\sigma}(\nabla u)-\boldsymbol{\sigma}\left(\nabla u_{L, h}\right), \nabla v\right)}{\|\nabla v\|_{p}} .
$$

Although the error measure $J_{u}\left(u_{L, h}\right)$ is not equivalent to the energy error $\left\|u-u_{L, h}\right\|_{V}$, we observe that under the assumption that $u_{L, h}$ is uniformly bounded in $V$, the monotonicity and Hölder continuity properties of the operator $A$ readily imply that $J_{u}\left(u_{L, h}\right) \rightarrow 0$ if and only if $\left\|u-u_{L, h}\right\|_{V} \rightarrow 0$. 
3.1. Abstract a posteriori error estimate distinguishing the discretization and linearization errors. Following [12], we now distinguish the discretization and linearization errors:

Theorem 3.1 (Abstract a posteriori error estimate distinguishing the discretization and linearization errors). Let $u \in V$ be the solution of (1.3) and let $u_{L, h} \in V_{h}$ be arbitrary. Then,

$$
\mathcal{J}_{u}\left(u_{L, h}\right) \leq\left\|f-A_{L} u_{L, h}\right\|_{V^{\prime}}+\left\|A_{L} u_{L, h}-A u_{L, h}\right\|_{V^{\prime}} .
$$

Proof. Since $A u=f$ in $V^{\prime}$, we infer that

$$
\left\|A u-A u_{L, h}\right\|_{V^{\prime}}=\left\|f-A u_{L, h}\right\|_{V^{\prime}}=\left\|f \pm A_{L} u_{L, h}-A u_{L, h}\right\|_{V^{\prime}},
$$

and we conclude using the triangle inequality.

Remark 3.2 (Discretization and linearization errors). Following [12], we call the first term in the righthand side of (3.2) the discretization error and the second one the linearization error. Since $A_{L} u_{L}=f$, where $u_{L}$ is the solution to the continuous linearized problem (2.11), the discretization error can also be written as $\left\|A_{L} u_{L}-A_{L} u_{L, h}\right\|_{V^{\prime}}$.

Remark 3.3 (Arbitrary $u_{L, h}$ ). In the statement of Theorem 3.1, the discrete function $u_{L, h}$ need not be the solution of the discrete linearized problem (2.12), but can instead be arbitrary in $V_{h}$. The same remark holds for the statement of Theorem 3.5 below.

3.2. Guaranteed and fully computable a posteriori error estimate. To define our a posteriori error estimate, we suppose the following:

Assumption 3.4 (Local conservation). There exists a vector field $\mathbf{t}_{h} \in \mathbf{W}^{q}(\operatorname{div}, \Omega):=\left\{\mathbf{v} \in \mathbf{L}^{q}(\Omega) ; \nabla\right.$. $\left.\mathbf{v} \in \mathbf{L}^{q}(\Omega)\right\}$ such that

$$
\left(\nabla \cdot \mathbf{t}_{h}, 1\right)_{D}=(f, 1)_{D} \quad \forall D \in \mathcal{D}_{h}^{\text {int }} .
$$

For each $D \in \mathcal{D}_{h}$, let the residual estimator $\eta_{\mathrm{R}, D}$, the diffusive flux estimator $\eta_{\mathrm{DF}, D}$, and the linearization estimator $\eta_{\mathrm{L}, D}$ be defined as

$$
\begin{aligned}
\eta_{\mathrm{R}, D} & :=m_{D}\left\|f-\nabla \cdot \mathbf{t}_{h}\right\|_{q, D}, \\
\eta_{\mathrm{DF}, D} & :=\left\|\boldsymbol{\sigma}_{L}\left(\nabla u_{L, h}\right)+\mathbf{t}_{h}\right\|_{q, D}, \\
\eta_{\mathrm{L}, D} & :=\left\|\boldsymbol{\sigma}\left(\nabla u_{L, h}\right)-\boldsymbol{\sigma}_{L}\left(\nabla u_{L, h}\right)\right\|_{q, D} .
\end{aligned}
$$

Here, $m_{D}=C_{\mathrm{P}, p, D} h_{D}$ if $D \in \mathcal{D}_{h}^{\text {int }}$ and $m_{D}=C_{\mathrm{F}, p, D, \partial \Omega} h_{D}$ if $D \in \mathcal{D}_{h}^{\text {ext }}$ and $C_{\mathrm{P}, p, D}$ is the constant from the generalized Poincaré inequality

$$
\left\|\varphi-\varphi_{D}\right\|_{p, D} \leq C_{\mathrm{P}, p, D} h_{D}\|\nabla \varphi\|_{p, D} \quad \forall \varphi \in W^{1, p}(D),
$$

$D \in \mathcal{D}_{h}^{\text {int }}$ (here $\left.\varphi_{D}=(\varphi, 1)_{D} /|D|\right)$ and $C_{\mathrm{F}, p, D, \partial \Omega}$ is the constant from the generalized Friedrichs inequality

$$
\|\varphi\|_{p, D} \leq C_{\mathrm{F}, p, D, \partial \Omega} h_{D}\|\nabla \varphi\|_{p, D} \quad \forall \varphi \in W^{1, p}(D) \text { such that } \varphi=0 \text { on } \partial \Omega \cap \partial D,
$$

$D \in \mathcal{D}_{h}^{\text {ext }}$. If $p=2, C_{\mathrm{P}, p, D}=1 / \pi$ if $D$ is convex and $C_{\mathrm{F}, p, D, \partial \Omega}=1$ in general, cf. [40] and the references therein. For $p \geq 2, C_{\mathrm{P}, p, D}=\pi^{-2 / p} d^{\frac{1}{2}-\frac{1}{p}}$ if $D$ is convex, see [32]. Note, however, that using the construction of $\mathbf{t}_{h}$ by (5.6a)-(5.6b) from Section 5 below, the values of these constants are actually not needed whenever $f$ is piecewise constant. We can now state the main result of this section:

Theorem 3.5 (A posteriori error estimate). Let $u \in V$ be the solution of (1.3) and let $u_{L, h} \in V_{h}$ be arbitrary. Let the error estimators $\eta_{\mathrm{R}, D}, \eta_{\mathrm{DF}, D}$, and $\eta_{\mathrm{L}, D}$ be given by (3.4)-(3.6). Then, under Assumption 3.4, there holds

$$
\mathcal{J}_{u}\left(u_{L, h}\right) \leq \eta:=\left\{\sum_{D \in \mathcal{D}_{h}}\left(\eta_{\mathrm{R}, D}+\eta_{\mathrm{DF}, D}\right)^{q}\right\}^{1 / q}+\left\{\sum_{D \in \mathcal{D}_{h}} \eta_{\mathrm{L}, D}^{q}\right\}^{1 / q} .
$$


Proof. We estimate the two terms in the right-hand side of (3.2).

(i) Estimate on the discretization error. Observe that for all $v \in V$ with $\|v\|_{V}=1$, there holds

$$
\left\langle f-A_{L} u_{L, h}, v\right\rangle_{V^{\prime}, V}=\left\langle f \pm \nabla \cdot \mathbf{t}_{h}-A_{L} u_{L, h}, v\right\rangle_{V^{\prime}, V}=\left\langle f-\nabla \cdot \mathbf{t}_{h}, v\right\rangle_{V^{\prime}, V}+\left\langle\nabla \cdot \mathbf{t}_{h}-A_{L} u_{L, h}, v\right\rangle_{V^{\prime}, V} .
$$

The first term is bounded as follows,

$$
\begin{aligned}
\left\langle f-\nabla \cdot \mathbf{t}_{h}, v\right\rangle_{V^{\prime}, V} & =\left(f-\nabla \cdot \mathbf{t}_{h}, v\right)=\sum_{D \in \mathcal{D}_{h}^{\text {int }}}\left(f-\nabla \cdot \mathbf{t}_{h}, v\right)_{D}+\sum_{D \in \mathcal{D}_{h}^{\text {ext }}}\left(f-\nabla \cdot \mathbf{t}_{h}, v\right)_{D} \\
& =\sum_{D \in \mathcal{D}_{h}^{\text {int }}}\left(f-\nabla \cdot \mathbf{t}_{h}, v-v_{D}\right)_{D}+\sum_{D \in \mathcal{D}_{h}^{\text {ext }}}\left(f-\nabla \cdot \mathbf{t}_{h}, v\right)_{D} \\
& \leq \sum_{D \in \mathcal{D}_{h}^{\text {int }}}\left\|f-\nabla \cdot \mathbf{t}_{h}\right\|_{q, D}\left\|v-v_{D}\right\|_{p, D}+\sum_{D \in \mathcal{D}_{h}^{\text {ext }}}\left\|f-\nabla \cdot \mathbf{t}_{h}\right\|_{q, D}\|v\|_{p, D} \\
& \leq \sum_{D \in \mathcal{D}_{h}} m_{D}\left\|f-\nabla \cdot \mathbf{t}_{h}\right\|_{q, D}\|\nabla v\|_{p, D}=\sum_{D \in \mathcal{D}_{h}} \eta_{\mathrm{R}, D}\|\nabla v\|_{p, D},
\end{aligned}
$$

where we have used the Hölder inequality together with (3.7) and (3.8). Furthermore, for the second term, using the Green theorem, definition (2.13) of the linearized operator, and the Hölder inequality yields

$$
\left\langle\nabla \cdot \mathbf{t}_{h}-A_{L} u_{L, h}, v\right\rangle_{V^{\prime}, V}=-\left(\mathbf{t}_{h}+\boldsymbol{\sigma}_{L}\left(\nabla u_{L, h}\right), \nabla v\right) \leq \sum_{D \in \mathcal{D}_{h}} \eta_{\mathrm{DF}, D}\|\nabla v\|_{p, D} .
$$

Collecting the two above bounds leads to

$$
\left\langle f-A_{L} u_{L, h}, v\right\rangle_{V^{\prime}, V} \leq \sum_{D \in \mathcal{D}_{h}}\left(\eta_{\mathrm{R}, D}+\eta_{\mathrm{DF}, D}\right)\|\nabla v\|_{p, D},
$$

whence the Hölder inequality yields

$$
\left\|f-A_{L} u_{L, h}\right\|_{V^{\prime}}=\sup _{v \in V,\|v\|_{V}=1}\left\langle f-A_{L} u_{L, h}, v\right\rangle_{V^{\prime}, V} \leq\left\{\sum_{D \in \mathcal{D}_{h}}\left(\eta_{\mathrm{R}, D}+\eta_{\mathrm{DF}, D}\right)^{q}\right\}^{1 / q} .
$$

(ii) Estimate on the linearization error. For all $v \in V$ with $\|v\|_{V}=1$, using definitions (2.1) and (2.13) along with the Hölder inequality yields

$$
\left\langle A_{L} u_{L, h}-A u_{L, h}, v\right\rangle_{V^{\prime}, V}=\left(\sigma_{L}\left(\nabla u_{L, h}\right)-\sigma\left(\nabla u_{L, h}\right), \nabla v\right) \leq\left\{\sum_{D \in \mathcal{D}_{h}} \eta_{\mathrm{L}, D}^{q}\right\}^{1 / q},
$$

which completes the proof.

3.3. Balancing discretization and linearization errors. We are now in a position to specify the stopping criterion for the iterative loop outlined in Section 2.3.

3.3.1. Global stopping criterion. Choose a positive parameter $\gamma$ and stop the iterative loop whenever

$$
\eta_{\mathrm{L}}:=\left\{\sum_{D \in \mathcal{D}_{h}} \eta_{\mathrm{L}, D}^{q}\right\}^{1 / q} \leq \gamma\left\{\sum_{D \in \mathcal{D}_{h}}\left(\eta_{\mathrm{R}, D}+\eta_{\mathrm{DF}, D}\right)^{q}\right\}^{1 / q}=: \gamma \eta_{\mathrm{D}} .
$$

This criterion equilibrates the global linearization estimator $\eta_{\mathrm{L}}$ and the global discretization estimator $\eta_{\mathrm{D}}$, up to the constant $\gamma$. It is global since it is based on quantities defined for all mesh elements. As we will see in Theorem 4.8 below, it is sufficient for proving global efficiency. 
3.3.2. Local stopping criterion. Choose a family of positive parameters $\left\{\gamma_{D}\right\}_{D \in \mathcal{D}_{h}}$ and stop the iterative loop whenever

$$
\eta_{\mathrm{L}, D} \leq \gamma_{D}\left(\eta_{\mathrm{R}, D}+\eta_{\mathrm{DF}, D}\right) \quad \forall D \in \mathcal{D}_{h}
$$

This criterion equilibrates the local linearization estimators $\eta_{\mathrm{L}, D}$ and the local discretization estimators $\eta_{\mathrm{R}, D}+\eta_{\mathrm{DF}, D}$, up to the constants $\gamma_{D}$. It is local since it is based on quantities defined for each mesh element separately. As we will see in Theorem 4.4 below, it implies local efficiency and hence suitability of our a posteriori error estimates to adaptive mesh refinement.

\section{EFFiCiency OF the estimators}

We examine in this section the local and global efficiencies of our estimates.

4.1. Preliminaries. Henceforth, we set for convenience

$$
\sigma_{L, h}:=\sigma_{L}\left(\nabla u_{h}\right)
$$

Taking into account the definitions (2.14) or (2.15) of the linearized flux function $\boldsymbol{\sigma}_{L}$ and recalling that both $u_{h}$ and the function $u_{0}$ at which the linearization is performed are piecewise affine, it is inferred that $\sigma_{L, h} \in\left[\mathbb{P}_{0}\left(\mathcal{T}_{h}\right)\right]^{d}$. The results presented in this section are valid more generally under the assumption $\boldsymbol{\sigma}_{L, h} \in\left[\mathbb{P}_{k}\left(\mathcal{T}_{h}\right)\right]^{d}$ for some fixed polynomial degree $k$. We also assume in this section that $f \in \mathbb{P}_{k}\left(\mathcal{T}_{h}\right)$.

In the sequel, $A \lesssim B$ stands for the inequality $A \leq C B$ with a generic constant $C$ independent of the mesh size $h$, the nonlinear and linearized functions $a$ and $a_{L}$, and the Lebesgue exponent $p$, but that can depend on the shape regularity parameter $\kappa$ of the mesh family $\left(\mathcal{S}_{h}\right)_{h}$ and on the polynomial degree $k$.

Assumption 4.1 (Approximation property of the reconstructed flux). The reconstructed flux $\mathbf{t}_{h}$ is in $\left[\mathbb{P}_{k}\left(\mathcal{T}_{h}\right)\right]^{d}$ and there holds for all $D \in \mathcal{D}_{h}$,

$$
\eta_{\mathrm{DF}, D} \lesssim \eta_{\mathrm{res}, D}:=\left\{\sum_{T \in \mathcal{S}_{D}} h_{T}^{q}\left\|f+\nabla \cdot \boldsymbol{\sigma}_{L, h}\right\|_{q, T}^{q}+\sum_{F \in \mathcal{G}_{D}^{\mathcal{T}}} h_{F}\left\|\llbracket \boldsymbol{\sigma}_{L, h} \cdot \mathbf{n} \rrbracket\right\|_{q, F}^{q}\right\}^{\frac{1}{q}},
$$

where $\mathcal{S}_{D} \subset \mathcal{S}_{h}$ collects the simplices of $\mathcal{S}_{h}$ included in $D$, and $\mathcal{G}_{D}^{\mathcal{T}} \subset \mathcal{G}_{h}^{\mathcal{T}}$ collects the faces of $\mathcal{G}_{h}^{\mathcal{T}}$ included in D; moreover, $\llbracket \cdot \rrbracket$ denotes the jump across a face.

We will verify Assumption 4.1 in Section 5 below. Under this assumption, we now prove a simple result, relating our estimates to classical residual ones; see, e.g., [31].

Lemma 4.2 (Upper bound by residual estimators). Under Assumption 4.1, there holds

$$
\eta_{\mathrm{R}, D}+\eta_{\mathrm{DF}, D} \lesssim \eta_{\mathrm{res}, D} \quad \forall D \in \mathcal{D}_{h}
$$

Proof. Taking into account (4.2), it remains to show the bound on $\eta_{\mathrm{R}, D}$. Since $h_{D} \lesssim h_{T}$ for all $T \in \mathcal{S}_{D}$, there holds

$$
\eta_{\mathrm{R}, D} \lesssim h_{D}\left\{\sum_{T \in \mathcal{S}_{D}}\left\|f-\nabla \cdot \mathbf{t}_{h}\right\|_{q, T}^{q}\right\}^{\frac{1}{q}} \lesssim\left\{\sum_{T \in \mathcal{S}_{D}} h_{T}^{q}\left\|f-\nabla \cdot \mathbf{t}_{h}\right\|_{q, T}^{q}\right\}^{\frac{1}{q}} .
$$

As a result, using the triangle inequality and the inverse inequality (A.1) with $k=1$ and $r=q$ leads to

$$
\begin{aligned}
\eta_{\mathrm{R}, D} & \lesssim\left\{\sum_{T \in \mathcal{S}_{D}} h_{T}^{q}\left\|f+\nabla \cdot \boldsymbol{\sigma}_{L, h}\right\|_{q, T}^{q}\right\}^{\frac{1}{q}}+\left\{\sum_{T \in \mathcal{S}_{D}} h_{T}^{q}\left\|\nabla \cdot\left(\boldsymbol{\sigma}_{L, h}+\mathbf{t}_{h}\right)\right\|_{q, T}^{q}\right\}^{\frac{1}{q}} \\
& \lesssim\left\{\sum_{T \in \mathcal{S}_{D}} h_{T}^{q}\left\|f+\nabla \cdot \boldsymbol{\sigma}_{L, h}\right\|_{q, T}^{q}\right\}^{\frac{1}{q}}+\eta_{\mathrm{DF}, D},
\end{aligned}
$$

whence the result readily follows. 
4.2. Local efficiency. We address here the local efficiency of the estimators of Theorem 3.5. We first give a result relying on the techniques presented in [35]. The proof is postponed to Section A.2.

Lemma 4.3 (Local efficiency of residual estimators). For all $D \in \mathcal{D}_{h}$, there holds

$$
\eta_{\mathrm{res}, D} \lesssim\left\|\boldsymbol{\sigma}(\nabla u)-\boldsymbol{\sigma}\left(\nabla u_{L, h}\right)\right\|_{q, D}+\eta_{\mathrm{L}, D} .
$$

We are now ready to announce and prove the main result of this section.

Theorem 4.4 (Local efficiency). Let (3.11), with $\gamma_{D}$ small enough, and (4.2) hold true. Then, for all $D \in \mathcal{D}_{h}$,

$$
\eta_{\mathrm{L}, D}+\eta_{\mathrm{R}, D}+\eta_{\mathrm{DF}, D} \lesssim\left\|\boldsymbol{\sigma}(\nabla u)-\boldsymbol{\sigma}\left(\nabla u_{L, h}\right)\right\|_{q, D} .
$$

Proof. Using (3.11), (4.3), and (4.4),

$$
\begin{aligned}
\eta_{\mathrm{L}, D}+\eta_{\mathrm{R}, D}+\eta_{\mathrm{DF}, D} & \leq\left(1+\gamma_{D}\right)\left(\eta_{\mathrm{R}, D}+\eta_{\mathrm{DF}, D}\right) \leq C\left(1+\gamma_{D}\right) \eta_{\mathrm{res}, D} \\
& \leq \tilde{C}\left(1+\gamma_{D}\right)\left(\left\|\boldsymbol{\sigma}(\nabla u)-\boldsymbol{\sigma}\left(\nabla u_{L, h}\right)\right\|_{q, D}+\eta_{\mathrm{L}, D}\right) \\
& \leq \tilde{C}\left(1+\gamma_{D}\right)\left\|\boldsymbol{\sigma}(\nabla u)-\boldsymbol{\sigma}\left(\nabla u_{L, h}\right)\right\|_{q, D}+\tilde{C}\left(1+\gamma_{D}\right) \gamma_{D}\left(\eta_{\mathrm{R}, D}+\eta_{\mathrm{DF}, D}\right) .
\end{aligned}
$$

Thus, it suffices to choose $\gamma_{D}=\min (1,1 /(2 \tilde{C}))$ in order to obtain

$$
\eta_{\mathrm{L}, D}+\eta_{\mathrm{R}, D}+\eta_{\mathrm{DF}, D} \leq 2 \tilde{C}\left(1+\gamma_{D}\right)\left\|\boldsymbol{\sigma}(\nabla u)-\boldsymbol{\sigma}\left(\nabla u_{L, h}\right)\right\|_{q, D} .
$$

Remark 4.5 (Local efficiency). Whereas the estimates are derived for the error measure $\mathcal{J}_{u}\left(u_{L, h}\right)$, the local efficiency of Theorem 4.4 uses the $L^{q}$-norm of the difference of fluxes. This is not fully satisfactory, but it seems to be the price to obtain local efficiency and not only global, as presented in the next section.

Remark 4.6 (Local efficiency on the given element only). Owing to the fact that only (a subset of) the interior faces of $\mathcal{S}_{D}$ appear(s) in (4.2), the local efficiency result (4.5) is stated on the given dual volume $D$ only (no neighbors appear). This is in correspondence with the fact that the stopping criterion (3.11) is also purely local.

4.3. Global efficiency. We address here the global efficiency of the estimators of Theorem 3.5, relying on the techniques presented in [35] and [36]. The proof is postponed to Section A.3.

Lemma 4.7 (Global efficiency of residual estimators). There holds

$$
\eta_{\mathrm{res}}:=\left\{\sum_{T \in \mathcal{S}_{h}} h_{T}^{q}\left\|f+\nabla \cdot \boldsymbol{\sigma}_{L, h}\right\|_{q, T}^{q}+\sum_{F \in \mathcal{G}_{h}^{T}} h_{F}\left\|\llbracket \boldsymbol{\sigma}_{L, h} \cdot \mathbf{n} \rrbracket\right\|_{q, F}^{q}\right\}^{\frac{1}{q}} \lesssim\left\|A u-A u_{L, h}\right\|_{V^{\prime}}+\eta_{\mathrm{L}} .
$$

We are now ready to announce the main result of this section. The proof is skipped since it follows by the same arguments as in Theorem 4.4.

Theorem 4.8 (Global efficiency). Let (3.10), with $\gamma$ small enough, and (4.2) hold true. Then,

$$
\eta \lesssim \mathcal{J}_{u}\left(u_{L, h}\right)
$$

Remark 4.9 (Robustness). Theorem 4.8 means that the a posteriori error estimates of Theorem 3.5 are fully robust with respect to the "size" of the nonlinearity. Indeed, the lower bound of Theorem 4.8 is independent of the nonlinear function a, as well as of the Lebesgue exponent $p$. 


\section{FluX ReCONSTRUCTION}

In this section, we give two examples for the reconstructed flux $\mathbf{t}_{h}$ satisfying Assumptions 3.4 and 4.1 . In both cases, the reconstructed flux $\mathbf{t}_{h}$ belongs to the lowest-order Raviart-Thomas-Nédélec finite element space $\mathbf{R T N}\left(\mathcal{S}_{h}\right)$ associated with the simplicial submesh $\mathcal{S}_{h}$.

Recalling the notation $\boldsymbol{\sigma}_{L, h}:=\boldsymbol{\sigma}_{L}\left(\nabla u_{L, h}\right)$, the finite element approximation to the continuous linearized problem (2.11), namely (2.12), can be written in the form

$$
\left(\boldsymbol{\sigma}_{L, h}, \nabla v_{h}\right)=\left(f, v_{h}\right), \quad \forall v_{h} \in V_{h},
$$

and observe that by assumption $\boldsymbol{\sigma}_{L, h}$ is piecewise constant on $\mathcal{T}_{h}$. We first assume that $f$ is piecewise constant on $\mathcal{T}_{h}$. The general case is postponed to Remark 5.5 below. Under these assumptions, following [4, Lemma 3] (cf. also [40]), we infer that (5.1) is equivalent to finding $u_{L, h} \in V_{h}$ such that

$$
-\left(\boldsymbol{\sigma}_{L, h} \cdot \mathbf{n}_{D}, 1\right)_{\partial D}=(f, 1)_{D} \quad \forall D \in \mathcal{D}_{h}^{\text {int }},
$$

where $\mathbf{n}_{D}$ denotes the outward unit normal vector to $D$. The scheme defined by (5.2) is also nothing but the vertex-centered finite volume method for approximating (2.11). In the vertex-centered finite volume framework, $f$ does not need to be piecewise constant on $\mathcal{T}_{h}$.

The degrees of freedom of the reconstructed flux $\mathbf{t}_{h} \in \mathbf{R T N}\left(\mathcal{S}_{h}\right)$ are its normal components on all the faces $F \in \mathcal{G}_{h}$. In the sequel, for $F \in \mathcal{G}_{h}, \mathbf{n}_{F}$ denotes a unit normal vector to $F$ (its orientation is fixed but irrelevant). We first set

$$
\mathbf{t}_{h} \cdot \mathbf{n}_{F}:=-\boldsymbol{\sigma}_{L, h} \cdot \mathbf{n}_{F} \quad \forall F \in \mathcal{G}_{h}^{\mathcal{D}} .
$$

An immediate and important consequence of (5.2) and (5.3) is

Lemma 5.1 (Local conservation). Assumption 3.4 holds true.

Following [40], there exist several ways of defining $\mathbf{t}_{h} \cdot \mathbf{n}_{F}$ on the remaining faces of $\mathcal{G}_{h}$ (that is, those located inside some $D \in \mathcal{D}_{h}$ and those located on the boundary $\partial \Omega$ ). We present here two methods.

- Direct prescription: Firstly, we can directly prescribe

$$
\mathbf{t}_{h} \cdot \mathbf{n}_{F}:=-\left\{\left\{\boldsymbol{\sigma}_{L, h} \cdot \mathbf{n}_{F}\right\}\right\}
$$

on all the remaining faces. Here, $\{\{\cdot\}$ denotes the mean-value on interior faces and the actual value on boundary faces.

- Prescription by local linear system solves: Secondly, following [5, 18, 40], we can solve local Neumann problems: for a given $D \in \mathcal{D}_{h}$, let

$$
\mathbf{R T N}_{\mathrm{N}}\left(\mathcal{S}_{D}\right)=\left\{\mathbf{v}_{h} \in \mathbf{R T N}\left(\mathcal{S}_{D}\right) ; \mathbf{v}_{h} \cdot \mathbf{n}_{F}=-\boldsymbol{\sigma}_{L, h} \cdot \mathbf{n}_{F} \quad \forall F \in \mathcal{G}_{h}^{\mathcal{D}}\right\} .
$$

Define $\mathbf{R T N}_{\mathrm{N}, 0}\left(\mathcal{S}_{D}\right)$ as $\mathbf{R T N}_{\mathrm{N}}\left(\mathcal{S}_{D}\right)$ but with the normal flux condition $\mathbf{v}_{h} \cdot \mathbf{n}_{F}=0$ for all $F \in \mathcal{G}_{h}^{\mathcal{D}}$. Let $\mathbb{P}_{0}^{*}\left(\mathcal{S}_{D}\right)$ be spanned by piecewise constants on $\mathcal{S}_{D}$ with zero mean on $D$ when $D \in \mathcal{D}_{h}^{\text {int }}$; when $D \in \mathcal{D}_{h}^{\text {ext }}$, the mean value condition is not imposed. The local problem consists in finding $\mathbf{t}_{h} \in \mathbf{R T N}_{\mathrm{N}}\left(\mathcal{S}_{D}\right)$ and $q_{h} \in \mathbb{P}_{0}^{*}\left(\mathcal{S}_{D}\right)$, the mixed finite element approximations (cf. [8]) of local Neumann problems on $D \in \mathcal{D}_{h}^{\text {int }}$ and local Neumann/Dirichlet problems on $D \in \mathcal{D}_{h}^{\text {ext }}$ :

$$
\begin{aligned}
\left(\mathbf{t}_{h}+\boldsymbol{\sigma}_{L, h}, \mathbf{v}_{h}\right)_{D}-\left(q_{h}, \nabla \cdot \mathbf{v}_{h}\right)_{D} & =0 & & \forall \mathbf{v}_{h} \in \mathbf{R T N}_{\mathrm{N}, 0}\left(\mathcal{S}_{D}\right), \\
\left(\nabla \cdot \mathbf{t}_{h}, \phi_{h}\right)_{D} & =\left(f, \phi_{h}\right)_{D} & & \forall \phi_{h} \in \mathbb{P}_{0}^{*}\left(\mathcal{S}_{D}\right) .
\end{aligned}
$$

Note in particular that the function $-\boldsymbol{\sigma}_{L, h} \cdot \mathbf{n}_{F}$, used to impose the Neumann boundary condition on $\partial D$ for each $D \in \mathcal{D}_{h}^{\text {int }}$, satisfies by (5.2) the compatibility condition with the datum $f$, whence the existence and uniqueness of the solution to (5.6a)-(5.6b).

Remark 5.2 (Comparison of the two approaches). A solution of a local linear system on each $D \in \mathcal{D}_{h}$ corresponding to (5.6a)-(5.6b) is necessary in the second case, but the advantage over the first case is twofold. Firstly, the effectivity indices are close to the optimal value of one, as observed in our numerical experiments. Secondly, owing to the assumption that $f$ is piecewise constant on $\mathcal{T}_{h}$ and to $(5.6 \mathrm{~b}), \| f-$ 
$\nabla \cdot \mathbf{t}_{h} \|_{q, T}=0$ for all $T \in \mathcal{S}_{h}$, so that $\eta_{\mathrm{R}, D}=0$ for all $D \in \mathcal{D}_{h}$ and one does not need to evaluate the constants $C_{\mathrm{P}, p, D}$ and $C_{\mathrm{F}, p, D, \partial \Omega}$ from (3.7) and (3.8), respectively. For more details, we refer to [40].

We now consider Assumption 4.1. The proof is given in Section A.4.

Lemma 5.3 (Approximation property). Assume that $\mathbf{t}_{h}$ is prescribed either directly through (5.4) or by solving the local problems (5.6a)-(5.6b). Then, Assumption 4.1 holds true.

Remark 5.4 (Other flux equilibration procedures). The procedure (5.6a)-(5.6b) is closely related to [27]. Many other approaches rely on equilibration on the given mesh $\mathcal{T}_{h}$. We cite in particular $[1,7,14,23]$. All these procedures can likewise be used to produce a discrete $\mathbf{W}^{q}(\operatorname{div}, \Omega)$-conforming vector field satisfying the local conservation property (Assumption 3.4). It is unknown to the authors whether these vector fields also satisfy the approximation property Assumption 4.1). If it is the case, then they can be used in the present framework. Following Remark 4.6, a slight modification of the stopping criterion (3.11) would be necessary for the approaches relying on the given mesh $\mathcal{T}_{h}$.

Remark 5.5 (General $f$ ). When $f$ is not piecewise constant on $\mathcal{T}_{h}$, we can proceed as follows: denote $f_{h} \in \mathbb{P}_{0}\left(\mathcal{T}_{h}\right)$ the function given by elementwise mean values of $f$. Consider $\bar{u}$, the solution to (1.3) with $f$ replaced by $f_{h}$, and $u_{L, h}$, the approximation of (2.12) with $f$ likewise replaced by $f_{h}$. Then, by the triangle inequality,

$$
\mathcal{J}_{u}\left(u_{L, h}\right)=\left\|A u-A u_{L, h}\right\|_{V^{\prime}} \leq\|A u-A \bar{u}\|_{V^{\prime}}+\left\|A \bar{u}-A u_{L, h}\right\|_{V^{\prime}} .
$$

The second term fits the framework of Sections 4 and 5 and is therefore bounded by the estimator $\eta$ (with $f$ replaced by $f_{h}$ ). The first term can be bounded as in the proof of Theorem 3.5 by the data oscillation term

$$
\eta_{\mathrm{Osc}}:=\left\{\sum_{T \in \mathcal{T}_{h}}\left(\left\|f-f_{h}\right\|_{q, T} C_{\mathrm{P}, p, T} h_{T}\right)^{q}\right\}^{1 / q} .
$$

Note that this term is localized on the mesh $\mathcal{T}_{h}$ and not on $\mathcal{D}_{h}$.

\section{Numerical RESUlts}

We present in this section numerical experiments for the $p$-Laplacian. We first recall that the error measure $\mathcal{J}_{u}\left(u_{L, h}\right)=\left\|A u-A u_{L, h}\right\|_{V^{\prime}}$ involves a dual norm that cannot be evaluated explicitly even if $u$ is known. Following [12], however, we deduce from (3.1) the following computable upper and lower bounds for $\mathcal{J}_{u}\left(u_{L, h}\right)$ :

$$
\begin{aligned}
& \mathcal{J}_{u}\left(u_{L, h}\right) \leq \mathcal{J}_{u}^{\text {up }}\left(u_{L, h}\right):=\left\|\boldsymbol{\sigma}(\nabla u)-\boldsymbol{\sigma}\left(\nabla u_{L, h}\right)\right\|_{q}, \\
& \mathcal{J}_{u}\left(u_{L, h}\right) \geq \mathcal{J}_{u}^{\text {low }}\left(u_{L, h}\right):=\frac{\left(\boldsymbol{\sigma}(\nabla u)-\boldsymbol{\sigma}\left(\nabla u_{L, h}\right), \nabla\left(u-u_{L, h}\right)\right)}{\left\|\nabla\left(u-u_{L, h}\right)\right\|_{p}} .
\end{aligned}
$$

As we will see below, our estimate $\eta$ defined in (3.9) turns out to be very close to $\mathcal{J}_{u}^{\text {up }}\left(u_{L, h}\right)$, whence our estimates are indeed very close to the error in the fluxes, which are often the physically most interesting quantity. We will use below the corresponding upper and lower effectivity indices, defined respectively as

$$
\mathcal{I}^{\text {up }}:=\frac{\eta}{\mathcal{J}_{u}^{\text {up }}\left(u_{L, h}\right)} \quad \text { and } \quad \mathcal{I}^{\text {low }}:=\frac{\eta}{\mathcal{J}_{u}^{\text {low }}\left(u_{L, h}\right)} .
$$

Consequently, the effectivity index for the error $\mathcal{J}_{u}\left(u_{L, h}\right)$, defined as

$$
\mathcal{I}:=\frac{\eta}{\mathcal{J}_{u}\left(u_{L, h}\right)},
$$

lies between $\mathcal{I}^{\text {up }}$ and $\mathcal{I}^{\text {low }}$. We observe that $\mathcal{I}^{\text {up }}$ can become smaller than one, which does not contradict that our estimates give a guaranteed upper bound; only $\mathcal{I}$ has to be greater than or equal to one.

We use continuous, piecewise affine finite elements and the Newton-Raphson method, which corresponds to solving (2.12) with $\boldsymbol{\sigma}_{L}\left(\nabla u_{L, h}\right)$ defined by (2.15). In order to construct the equilibrated flux 


\begin{tabular}{cccccccc}
\hline & \multicolumn{5}{c}{ flux } & & \multicolumn{2}{c}{ potential } \\
\cline { 2 - 4 } \cline { 7 - 8 }$p$ & regularity & error up & error low & estimate & & regularity & error energy \\
\hline 1.4 & $\mathbf{W}^{1.57, q}$ & 1.01 & 1.01 & 1.00 & & $W^{4.93, p}$ & 1.01 \\
3 & $\mathbf{W}^{2.33, q}$ & 1.01 & 1.01 & 1.01 & & $W^{2.17, p}$ & 1.00 \\
10 & $\mathbf{W}^{2.80, q}$ & 1.00 & 1.68 & 1.00 & & $W^{1.31, p}$ & 0.31 \\
\hline
\end{tabular}

TABLE 1. Flux and potential regularities and experimental orders of convergence, case 1

$\mathbf{t}_{h}$, we use the solution of local Neumann problems by the mixed finite element method (5.6a)-(5.6b). We use the local stopping criterion (3.11) with $\gamma_{D}=0.1$ for all $D \in \mathcal{D}_{h}$. On an initial mesh, we start the nonlinear iterative solver from the interpolation of the exact solution. On refined meshes, we interpolate the approximate solution from the next coarser mesh. We consider the following overall strategy:

(1) On the given mesh, perform the iterative linearization of Section 2.3 until the local stopping criterion (3.11) is satisfied;

(2) If $\eta$ is less than the desired precision, then stop, else refine the mesh adaptively, on the basis of $\eta$, and go to step (1).

In practice, all the elements where the estimate exceeds $50 \%$ of the maximal error are marked for refinement. The error stemming from inhomogeneous boundary conditions is not taken into account.

All the linear systems within the nonlinear iterative procedure are solved by a direct solver. The present approach can also be combined with a linear iterative solver, and to further save computational effort, the latter can be stopped whenever the algebraic error does not contribute significantly to the overall error, following [21].

6.1. A first test case. In this first test case, $\Omega:=] 0,1[\times] 0,1[, f=2$, and the Dirichlet boundary condition is given by the exact solution

$$
u(x, y)=-\frac{p-1}{p}|(x, y)-(0.5,0.5)|^{\frac{p}{p-1}}+\frac{p-1}{p}\left(\frac{1}{2}\right)^{\frac{p}{p-1}} .
$$

This is a two-dimensional extension of a test case from [11]. The Sobolev regularity for the potential $u$ and the flux $-\boldsymbol{\sigma}(\nabla u)$ is reported in Table 1 for the different values of $p$ considered in the experiments. More generally, $u \in W^{s_{u}, p}(\Omega)$ and $\boldsymbol{\sigma}(\nabla u) \in \mathbf{W}^{s_{\boldsymbol{\sigma}}, q}(\Omega):=\left[W^{s_{\boldsymbol{\sigma}}, q}(\Omega)\right]^{d}$ with $s_{u}<p /(p-1)+2 / p$ and $s_{\boldsymbol{\sigma}}<3-2 / p$. In particular, the flux is always sufficiently regular for all values of $p \in(1,+\infty)$ (that is, $s_{\boldsymbol{\sigma}}>1$ ), whereas the potential can be less regular than $W^{2, p}$ for values of $p$ larger than $\sim 3.73$. In Table 1, we also report the experimental orders of convergence

$$
\text { e.o.c. }:=\frac{\log \left(e_{N}\right)-\log \left(e_{N-1}\right)}{\frac{1}{2} \log \left|\mathcal{V}_{N-1}\right|-\frac{1}{2} \log \left|\mathcal{V}_{N}\right|},
$$

where $e_{N}$ is the error on the last mesh, $e_{N-1}$ is the error on the last but one mesh, and $\left|\mathcal{V}_{N}\right|$ and $\left|\mathcal{V}_{N-1}\right|$ denote the corresponding number of vertices. For the errors $e_{i}$, we consider the error upper bound $\mathcal{J}_{u}^{\text {up }}\left(u_{L, h}\right)$ from (6.1a), the error lower bound $\mathcal{J}_{u}^{\text {low }}\left(u_{L, h}\right)$ from $(6.1 \mathrm{~b})$, the total estimator $\eta$, and the energy error $\left\|\nabla\left(u-u_{L, h}\right)\right\|_{p}$. We only consider here uniformly refined meshes; since the flux $-\boldsymbol{\sigma}(\nabla u)$ is always regular and our a posteriori error estimates are related to the error in the flux, adaptive refinement does not lead here to improved convergence rates.

Figures 2-4 present the error upper and lower bounds $\mathcal{J}_{u}^{\text {up }}\left(u_{L, h}\right)$ and $\mathcal{J}_{u}^{\text {low }}\left(u_{L, h}\right)$, the total estimators $\eta$, and the corresponding upper and lower effectivity indices $\mathcal{I}^{\text {up }}$ and $\mathcal{I}^{\text {low }}$ as a function of the number of mesh vertices. We can see from Figures $2-4$ that $\mathcal{I}^{\text {up }}$ is very close to one for all the considered values of $p$. We have proven in Sections 3 and 4 that $\eta \sim \mathcal{J}_{u}\left(u_{L, h}\right)$, i.e., that $\eta$ and $\mathcal{J}_{u}\left(u_{L, h}\right)$ are equivalent up to a constant independent of the nonlinear function $a$ and of $p$. Thus, since numerically $\eta \sim \mathcal{J}_{u}^{\operatorname{up}}\left(u_{L, h}\right)$, we deduce that in the present case, $\mathcal{J}_{u}\left(u_{L, h}\right) \sim \mathcal{J}_{u}^{\text {up }}\left(u_{L, h}\right)$. This is not the case for the computable lower 

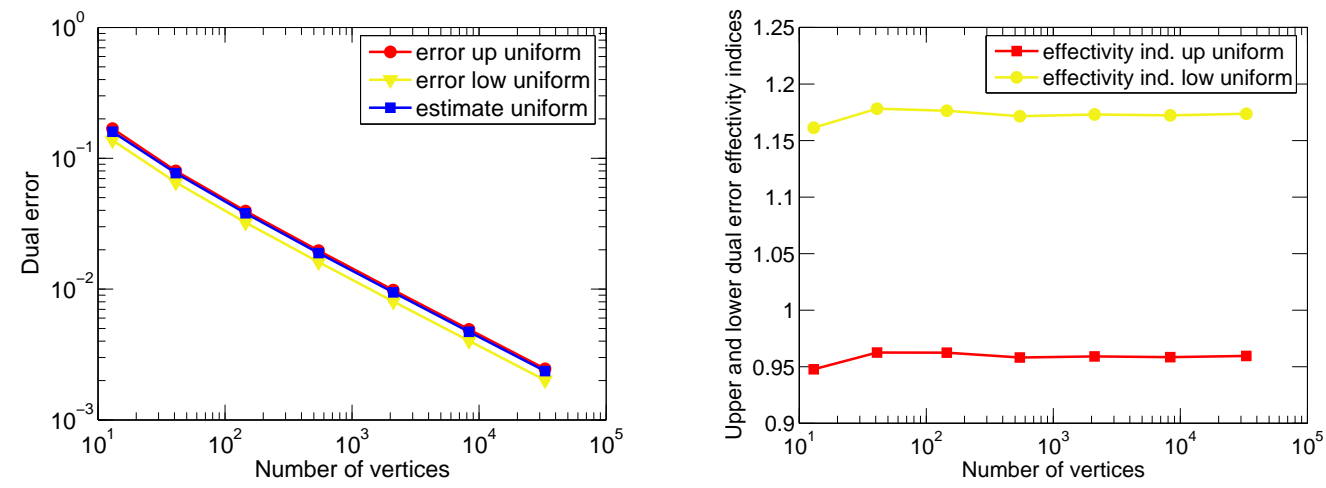

FIGURE 2. Estimated and actual errors (left) and corresponding effectivity indices (right) for $p=1.4$, case 1
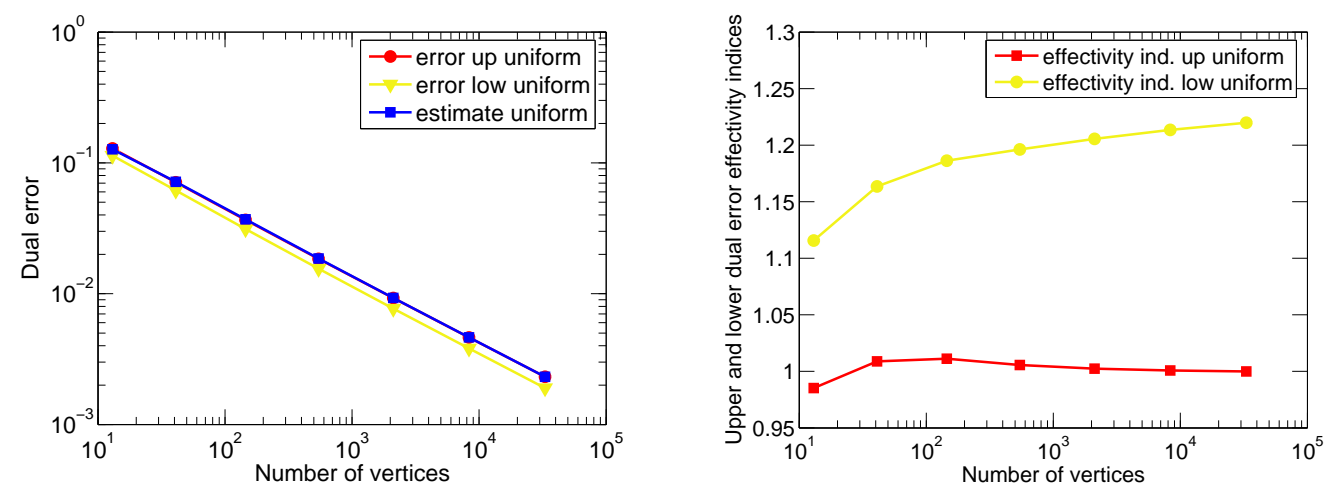

FIGURE 3. Estimated and actual errors (left) and corresponding effectivity indices (right) for $p=3$, case 1

bound $\mathcal{J}_{u}^{\text {low }}\left(u_{L, h}\right)$ for the larger values of $p$. The increase of the lower effectivity index $\mathcal{I}^{\text {low }}$ for $p=10$ (see Figure 4, right) can be attributed to the fact that in this case, the potential has low regularity, as reflected in Table 1 and that $\mathcal{J}_{u}^{\text {low }}\left(u_{L, h}\right)$ is scaled by the reciprocal of $\left\|\nabla\left(u-u_{L, h}\right)\right\|_{p}$.

Figure 5 compares the error distribution predicted by our estimator $\eta$ (we show the quantity $\eta_{\mathrm{R}, D}+$ $\eta_{\mathrm{DF}, D}+\eta_{\mathrm{L}, D}$ on each dual volume) with the actual error represented by the localized version of its upper bound, namely $\left\|\boldsymbol{\sigma}(\nabla u)-\boldsymbol{\sigma}\left(\nabla u_{L, h}\right)\right\|_{q, D}$. By Remark 5.2, $\eta_{\mathrm{R}, D}=0$ for all $D \in \mathcal{D}_{h}$ in the present case, while $\eta_{\mathrm{L}, D}$ is bounded by (3.11); hence, the only significative contribution to $\eta$ stems from the diffusive flux estimators $\eta_{\mathrm{DF}, D}$. We stress that all the above results are presented with the Newton-Raphson method not fully converged but stopped following (3.11) with $\gamma_{D}=0.1$.

Finally, Figures 6-7 illustrate the performance of our stopping criterion for the Newton-Raphson iteration by comparing it to a more classical stopping criterion, namely $\eta_{\mathrm{L}} \leq 10^{-8}$. Firstly, Figure 6 presents the discretization estimator $\eta_{\mathrm{D}}$, the linearization estimator $\eta_{\mathrm{L}}$, the total estimator $\eta$, and error upper bound $\mathcal{J}_{u}^{\text {up }}\left(u_{L, h}\right)$ as a function of Newton-Raphson iterations on a fixed mesh. We clearly see that the linearization estimator dominates the discretization one only at the first few iterations, and then becomes negligible while the total error stagnates. This confirms that the Newton-Raphson iteration can be safely stopped rather early. This effect becomes more pronounced as $p$ is increased and the mesh is refined. For instance, in the left part of Figure 6, the global stopping criterion (3.10) is reached after 5 iterations and the classical one after 11 iterations; in the right part of the figure, these numbers become 

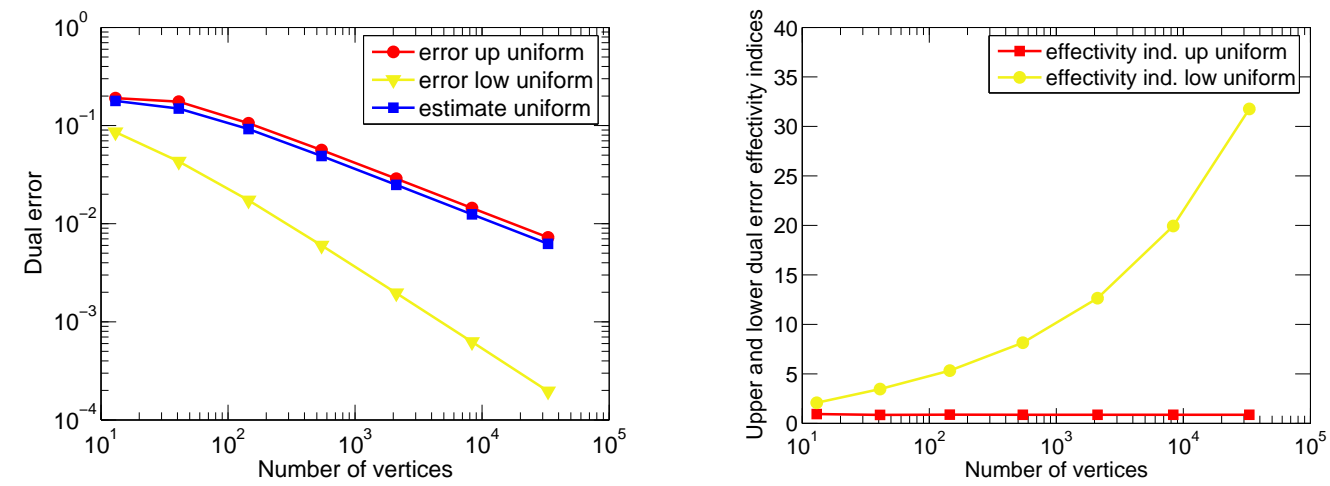

FIGURE 4. Estimated and actual errors (left) and corresponding effectivity indices (right) for $p=10$, case 1
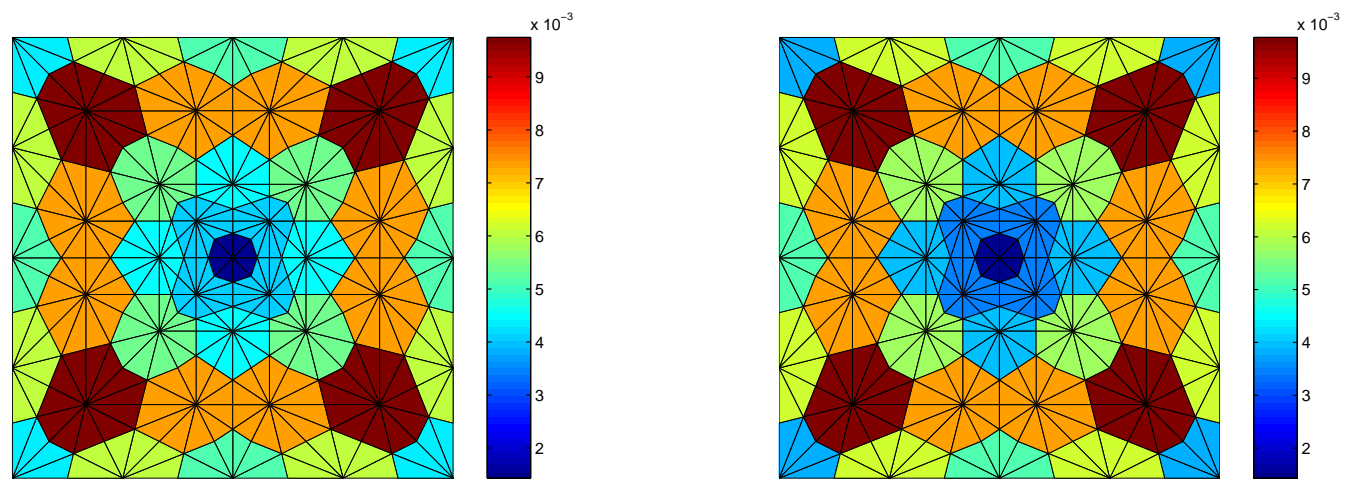

Figure 5. Estimated (left) and actual (right) error distribution for $p=3$, case 1

respectively 4 and 12. Secondly, the left part of Figure 7 presents the Newton-Raphson iteration numbers on a series of uniformly refined meshes in two situations, namely the global stopping criterion (3.10) and the classical one. It appears that around $50 \%$ of Newton-Raphson iterations can be spared. The advantage of the present algorithm shows more noticeably in the right part of Figure 7 . Here, we refine the mesh in an adaptive way. As we remarked before, this does not lead to increased experimental orders of convergence, as the flux possesses enough regularity. However, it appears that the elements slowing down the Newton-Raphson convergence are suitably refined, so that now the number of necessary iterations with the global stopping criterion (3.10) with $\gamma=0.1$ on refined meshes drops down to one or two. This should be compared with the upper curve in the left part of Figure 7, showing that the number of iterations using the classical stopping criterion in combination with uniform mesh refinement is about 10. A similar behavior is observed for the case $p=1.4$ (not shown). This effect is less pronounced for $p=3$ since in this case, the Newton-Raphson method always converges in a few iterations.

6.2. A second test case. We consider here a test case taken from [9, Example 3]. We consider the L-shaped domain $\Omega:=]-1,1[\times]-1,1[\backslash[0,1] \times[-1,0]$ and prescribe the Dirichlet boundary condition and the source term $f$ by the exact solution

$$
u(r, \theta)=r^{\alpha} \sin (\alpha \theta) .
$$



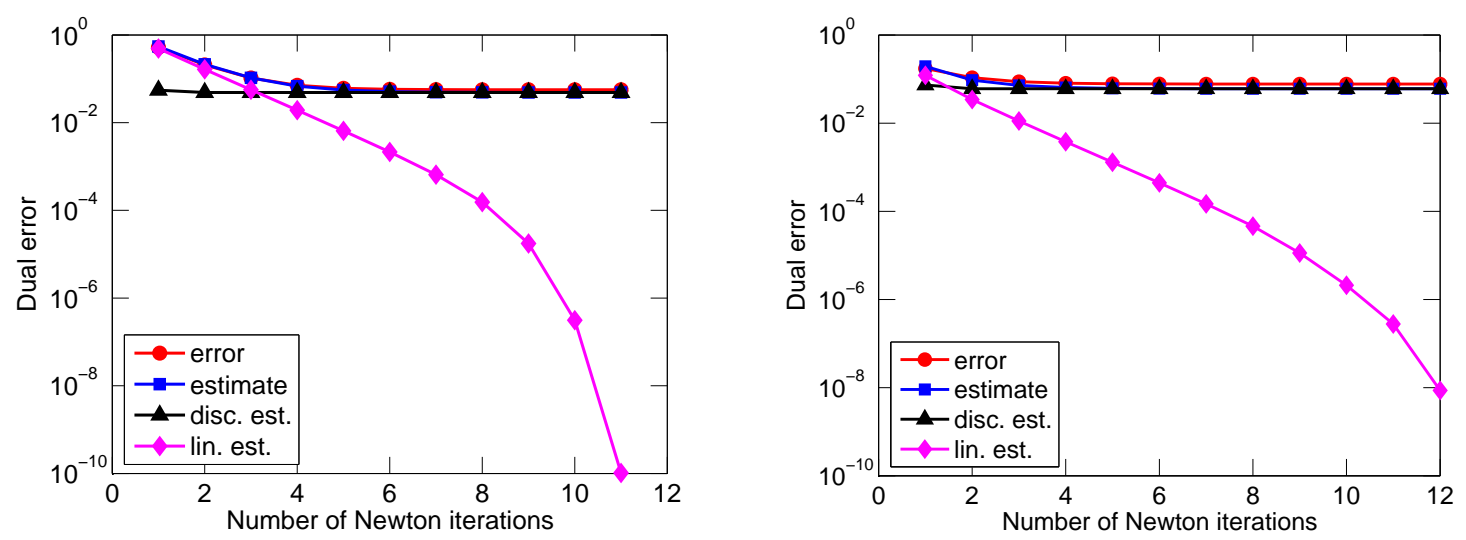

Figure 6. Discretization and linearization estimators, total estimator, and total error as a function of Newton-Raphson iterations for $p=10$ and the 4 th level uniformly refined mesh (left) and $p=50$ and the 5th level uniformly refined mesh (right), case 1
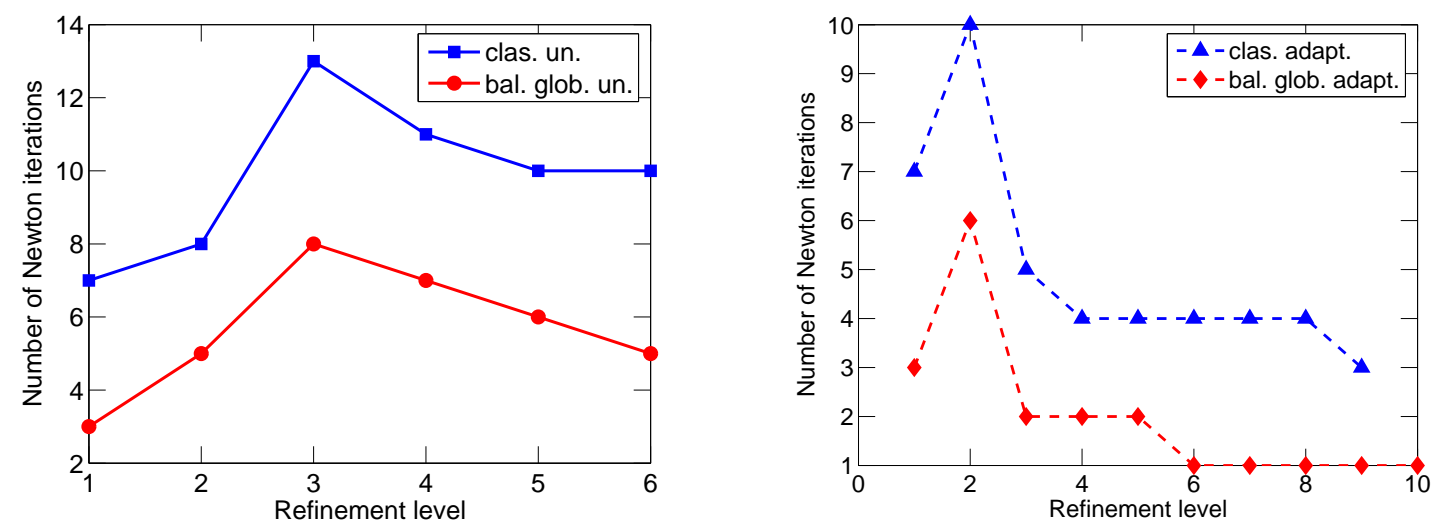

FiguRE 7. Number of Newton-Raphson iterations for the classical stopping criterion and the global stopping criterion (3.10) on uniformly (left)/adaptively (right) refined meshes, $p=10$, case 1

Here $(r, \theta)$ are the polar coordinates and $\alpha=7 / 8$. We consider the value $p=4$ for which the regularity of the potential and the flux, as defined above, are $s_{u}=1.38$ and $s_{\boldsymbol{\sigma}}=1.13$. The experimental orders of convergence are close to one for the error upper and lower bounds and for the error estimate using either uniform and adaptively refined meshes, while the experimental order of convergence for the potential energy norm is 0.38 on uniform meshes and 0.89 on adaptively refined meshes.

For this second test case, $f$ is not piecewise constant and hence, following Remark 5.5, the final a posteriori error estimate is given by $\eta+\eta_{\text {Osc }}$, with $f$ replaced by $f_{h}$ in $\eta$. The stopping criteria of Section 3.3 are not modified, that is, they rely on $\eta$ and its components only. Likewise, we only use $\eta$ and not $\eta+\eta_{\text {Osc }}$ to drive mesh adaptivity. Figure 8 presents the error upper and lower error bounds $\mathcal{J}_{u}^{\text {up }}\left(u_{L, h}\right)$ and $\mathcal{J}_{u}^{\text {low }}\left(u_{L, h}\right)$, the total estimators $\eta$, and the effectivity indices $\left(\eta+\eta_{\text {Osc }}\right) / \mathcal{J}_{u}^{\text {up }}\left(u_{L, h}\right)$ and $\left(\eta+\eta_{\text {Osc }}\right) / \mathcal{J}_{u}^{\text {low }}\left(u_{L, h}\right)$ as a function of the number of mesh vertices. In particular the effectivity indices $\left(\eta+\eta_{\text {Osc }}\right) / \mathcal{J}_{u}^{\text {up }}\left(u_{L, h}\right)$ are dominated by the data oscillation $\eta_{\text {Osc }} / \mathcal{J}_{u}^{\text {up }}\left(u_{L, h}\right)$ for rough meshes, since the source term $f$ is singular here. They only tend to the optimal value of one when $\eta_{\text {Osc }}$ becomes 

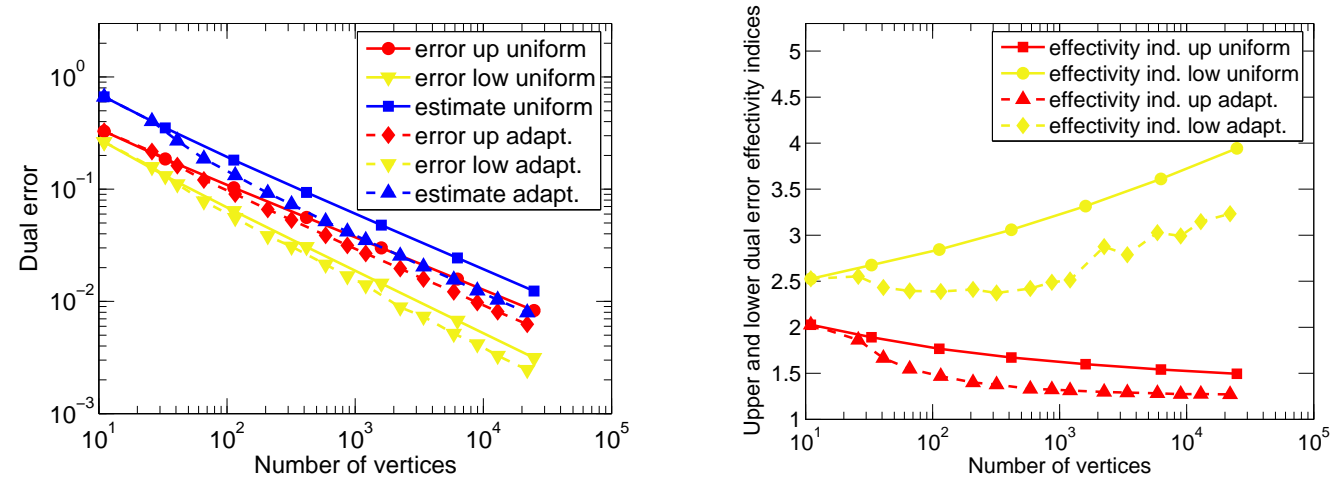

FIGURE 8. Estimated and actual errors (left) and corresponding effectivity indices (right) for $p=4$, case 2
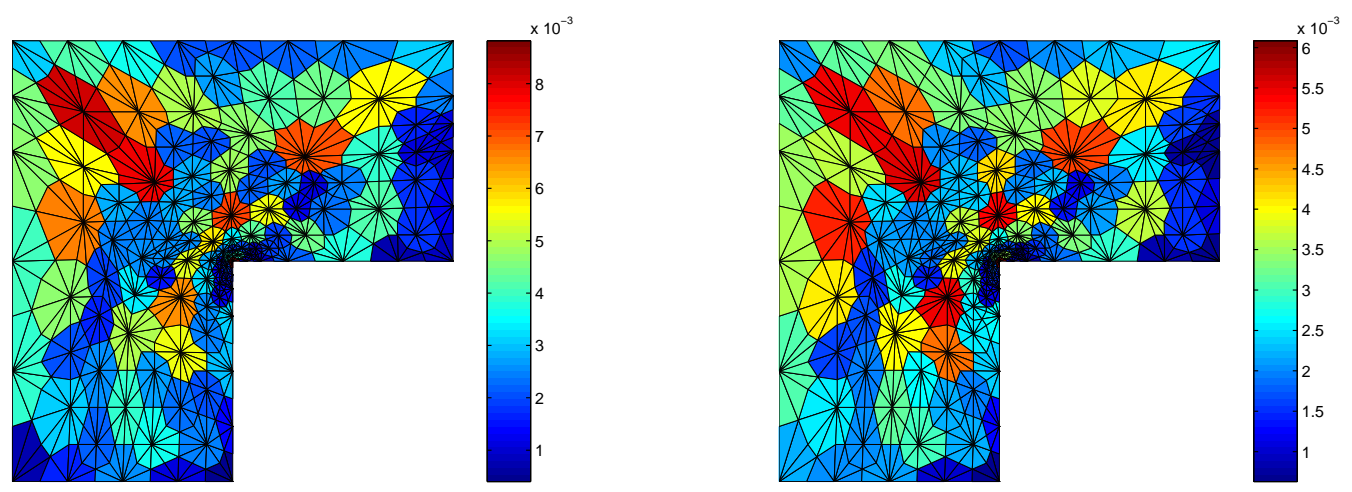

Figure 9. Estimated (left) and actual (right) error distribution for $p=4$, case 2

insignificant. The effectivity index $\eta / \mathcal{J}_{u}^{\text {up }}\left(u_{L, h}\right)$ is close to one on all meshes, uniformly or adaptively refined, in agreement with the theory and similarly to case 1. Finally, Figure 9 shows the comparison of the predicted error distribution given by our estimator $\eta$ and of the actual error, both quantities being localized as before, at the 5 th adaptively refined mesh.

\section{Appendix A. Technical Results}

In this appendix we collect various technical results. Henceforth, $C_{\kappa}$ denotes a generic constant that only depends on the shape-regularity parameter $\kappa$ of the mesh family $\left(\mathcal{S}_{h}\right)_{h}$ and whose value can change at each occurrence.

A.1. Inverse inequality. Let $T \in \mathcal{S}_{h}$ with diameter denoted by $h_{T}$. For an integer $k \geq 1$, let $\mathbb{P}_{k}(T)$ denote the vector space of polynomials of total degree $\leq k$ on $T$.

Lemma A.1. For all $v \in \mathbb{P}_{k}(T)$ and for all $r \in[1,+\infty]$, there holds

$$
\|\nabla v\|_{r, T} \leq C h_{T}^{-1}\|v\|_{r, T},
$$

where the constant $C$ can depend on $d, k$, and $\kappa$, but is independent of the Lebesgue exponent $r$. 
Proof. Following Verfürth [37], for a set $K \subset \Omega$ with positive $d$-dimensional measure, we consider the constants

$$
\begin{aligned}
C_{d, k, r}^{\sharp}(K) & :=\sup _{v \in \mathbb{P}_{k}(K) \backslash\{0\}} \frac{|K|^{\frac{1}{2}-\frac{1}{r}}\|v\|_{r, K}}{\|v\|_{K}}, \\
C_{d, k, r}^{b}(K) & :=\sup _{v \in \mathbb{P}_{k}(K) \backslash\{0\}} \frac{\|v\|_{K}}{|K|^{\frac{1}{2}-\frac{1}{r}}\|v\|_{r, K}},
\end{aligned}
$$

where $|K|$ denotes the $d$-dimensional Lebesgue measure of $K$. It is proven in [37] that

$$
\begin{aligned}
& C_{d, k, r}^{\sharp}(K) \leq \begin{cases}1 & \text { if } 1 \leq p \leq 2, \\
C_{d, k, \infty}^{\sharp}(K)^{1-\frac{2}{r}} & \text { if } 2<p \leq+\infty,\end{cases} \\
& C_{d, k, r}^{b}(K) \leq \begin{cases}C_{d, k, \infty}^{\sharp}(K)^{\frac{2}{r}-1} & \text { if } 1 \leq p \leq 2, \\
1 & \text { if } 2<p \leq+\infty,\end{cases}
\end{aligned}
$$

so that

$$
C_{d, k, r}^{b}(K) C_{d, k, r}^{\sharp}(K) \leq C_{d, k, \infty}^{\sharp}(K)^{\left|1-\frac{2}{r}\right|} .
$$

Moreover, for a $d$-dimensional simplex $T$, there holds

$$
C_{d, k, \infty}^{\sharp}(T) \leq C_{d, k}^{\star}:=(2 k+2)^{\frac{1}{2}}(4 k+2)^{\frac{d-1}{2}} .
$$

Hence, since $C_{d, k}^{\star} \geq 1$ and $\left|1-\frac{2}{r}\right| \leq 1$,

$$
C_{d, k, r}^{b}(T) C_{d, k, r}^{\sharp}(T) \leq C_{d, k}^{\star} .
$$

As a result, using the constant $C_{\kappa}$ from the usual inverse inequality in the $L^{2}$-setting [17] leads to

$$
\begin{aligned}
\|\nabla v\|_{r, T} & \leq C_{d, k, r}^{b}(T)|T|^{\frac{1}{r}-\frac{1}{2}}\|\nabla v\|_{T} \leq C_{\kappa} h_{T}^{-1} C_{d, k, r}^{b}(T)|T|^{\frac{1}{r}-\frac{1}{2}}\|v\|_{T} \\
& \leq C_{\kappa} h_{T}^{-1} C_{d, k, r}^{b}(T) C_{d, k, r}^{\sharp}(T)\|v\|_{r, T} \leq C_{d, k}^{\star} C_{\kappa} h_{T}^{-1}\|v\|_{r, T},
\end{aligned}
$$

completing the proof.

A.2. Proof of Lemma 4.3. We will use the following inequalities (see [33] for the $L^{2}$ framework and [35, 37] for the extension to the $L^{p}$ framework): for all $T \in \mathcal{S}_{h}$, for all $v \in \mathbb{P}_{k}(T)$, for all $F \subset \partial T$, and for all $\phi \in \mathbb{P}_{k}(F)$, there holds

$$
\begin{aligned}
\|v\|_{q, T} & \lesssim \sup _{w \in \mathbb{P}_{k}(T),\|w\|_{p, T}=1}\left(v, \Psi_{T} w\right)_{T}, \\
\left\|\nabla\left(\Psi_{T} v\right)\right\|_{p, T} & \lesssim h_{T}^{-1}\|v\|_{p, T}, \\
\|\phi\|_{q, F} & \lesssim \sup _{w \in \mathbb{P}_{k}(F),\|w\|_{p, F}=1}\left(\phi, \Psi_{F} w\right)_{F}, \\
\left\|\nabla\left(\Psi_{F} \phi\right)\right\|_{p, T} & \lesssim h_{F}^{-\frac{1}{q}}\|\phi\|_{p, F}, \\
\left\|\Psi_{F} \phi\right\|_{p, T} & \lesssim h_{F}^{\frac{1}{p}}\|\phi\|_{p, F},
\end{aligned}
$$

where $\Psi_{T}$ is the usual element bubble associated with $T$ and $\Psi_{F}$ the usual extension operator on the mesh $\mathcal{S}_{h}$ associated with the face bubble on $F$. The proof is decomposed into three parts. Let $D \in \mathcal{D}_{h}$.

We first prove that

$$
h_{T}\left\|f+\nabla \cdot \boldsymbol{\sigma}_{L, h}\right\|_{q, T} \lesssim\left\|\boldsymbol{\sigma}(\nabla u)-\boldsymbol{\sigma}_{L, h}\right\|_{q, T} \quad \forall T \in \mathcal{S}_{D} .
$$


Let $T \in \mathcal{S}_{D}$, set $v:=f+\nabla \cdot \boldsymbol{\sigma}_{L, h}$ and observe that $v \in \mathbb{P}_{k}(T)$. Thus, using (A.7a), (1.3), the Green theorem, the fact that $\Psi_{T}$ vanishes on $\partial T$, the Hölder inequality, and (A.7b) leads to

$$
\begin{aligned}
\|v\|_{q, T} & \lesssim \sup _{w \in \mathbb{P}_{k}(T),\|w\|_{p, T}=1}\left(f+\nabla \cdot \boldsymbol{\sigma}_{L, h}, \Psi_{T} w\right)_{T} \\
& =\sup _{w \in \mathbb{P}_{k}(T),\|w\|_{p, T}=1}\left(\boldsymbol{\sigma}(\nabla u)-\boldsymbol{\sigma}_{L, h}, \nabla\left(\Psi_{T} w\right)\right)_{T} \\
& \lesssim \sup _{w \in \mathbb{P}_{k}(T),\|w\|_{p, T}=1}\left\|\boldsymbol{\sigma}(\nabla u)-\boldsymbol{\sigma}_{L, h}\right\|_{q, T} h_{T}^{-1}\|w\|_{p, T} \\
& =h_{T}^{-1}\left\|\boldsymbol{\sigma}(\nabla u)-\boldsymbol{\sigma}_{L, h}\right\|_{q, T},
\end{aligned}
$$

whence (A.8) follows.

We next show that

$$
h_{F}^{\frac{1}{q}}\left\|\llbracket \boldsymbol{\sigma}_{L, h} \cdot \mathbf{n} \rrbracket\right\|_{q, F} \lesssim\left\|\boldsymbol{\sigma}(\nabla u)-\boldsymbol{\sigma}_{L, h}\right\|_{q, \omega_{F}} \quad \forall F \in \mathcal{G}_{D}^{\mathcal{T}},
$$

where $\omega_{F} \subset \Omega$ denotes the union of the two simplices of $\mathcal{S}_{h}$ sharing $F$. Note that $\cup_{F \in \mathcal{G}_{D}^{\mathcal{T}}} \omega_{F}$ forms a partition of the dual volume $D$. Set $\phi:=\llbracket \boldsymbol{\sigma}_{L, h} \cdot \mathbf{n} \rrbracket$ and observe that $\phi \in \mathbb{P}_{k}(F)$. Let $w \in \mathbb{P}_{k}(F)$ with $\|w\|_{p, F}=1$. Using (1.3), the Green theorem, the properties of the bubble functions, the Hölder inequality, (A.7d), (A.7e), (A.8), and the fact that $1 / q=1-1 / p$ then yields

$$
\begin{aligned}
\left(\phi, \Psi_{F} w\right)_{F} & =\left(f+\nabla \cdot \boldsymbol{\sigma}_{L, h}, \Psi_{F} w\right)_{\omega_{F}}-\left(\boldsymbol{\sigma}(\nabla u)-\boldsymbol{\sigma}_{L, h}, \nabla\left(\Psi_{F} w\right)\right)_{\omega_{F}} \\
& \leq\left\|f+\nabla \cdot \boldsymbol{\sigma}_{L, h}\right\|_{q, \omega_{F}}\left\|\Psi_{F} w\right\|_{p, \omega_{F}}+\left\|\boldsymbol{\sigma}(\nabla u)-\boldsymbol{\sigma}_{L, h}\right\|_{q, \omega_{F}}\left\|\nabla\left(\Psi_{F} w\right)\right\|_{p, \omega_{F}} \\
& \lesssim h_{F}^{\frac{1}{p}}\left\|f+\nabla \cdot \boldsymbol{\sigma}_{L, h}\right\|_{q, \omega_{F}}\|w\|_{p, F}+h_{F}^{-\frac{1}{q}}\left\|\boldsymbol{\sigma}(\nabla u)-\boldsymbol{\sigma}_{L, h}\right\|_{q, \omega_{F}}\|w\|_{p, F} \\
& \lesssim h_{F}^{-\frac{1}{q}}\left\|\boldsymbol{\sigma}(\nabla u)-\boldsymbol{\sigma}_{L, h}\right\|_{q, \omega_{F}}\|w\|_{p, F}=h_{F}^{-\frac{1}{q}}\left\|\boldsymbol{\sigma}(\nabla u)-\boldsymbol{\sigma}_{L, h}\right\|_{q, \omega_{F}},
\end{aligned}
$$

whence (A.9) follows from (A.7c).

Finally, combining the above results leads to $\eta_{\mathrm{res}, D} \lesssim\left\|\boldsymbol{\sigma}(\nabla u)-\boldsymbol{\sigma}_{L, h}\right\|_{q, D}$, and using the triangle inequality yields

whence (4.4) follows.

$$
\left\|\boldsymbol{\sigma}(\nabla u)-\boldsymbol{\sigma}_{L, h}\right\|_{q, D} \leq\left\|\boldsymbol{\sigma}(\nabla u)-\boldsymbol{\sigma}\left(\nabla u_{L, h}\right)\right\|_{q, D}+\eta_{\mathrm{L}, D}
$$

A.3. Proof of Lemma 4.7. We first give a technical extension of (A.7a). Let $\mathbb{P}_{k}\left(\mathcal{S}_{h}\right)$ be spanned by piecewise polynomials of total degree $\leq k$ on the mesh $\mathcal{S}_{h}$. Then, for all $v \in \mathbb{P}_{k}\left(\mathcal{S}_{h}\right)$,

$$
\left\{\sum_{T \in \mathcal{S}_{h}} h_{T}^{q}\|v\|_{q, T}^{q}\right\}^{\frac{1}{q}} \lesssim \sup _{w \in \mathbb{P}_{k}\left(\mathcal{S}_{h}\right),\|w\|_{p}=1} \sum_{T \in \mathcal{S}_{h}}\left(v, h_{T} \Psi_{T} w\right)_{T} .
$$

Indeed, for a given $T \in \mathcal{S}_{h}$, multiplying (A.7a) by $h_{T}$ yields

$$
\left\{h_{T}^{q}\|v\|_{q, T}^{q}\right\}^{\frac{1}{q}} \lesssim \sup _{w \in \mathbb{P}_{k}(T),\|w\|_{p, T}=1}\left(v, h_{T} \Psi_{T} w\right)_{T}
$$

Thus,

$$
h_{T}^{q}\|v\|_{q, T}^{q}=\left\{h_{T}^{q}\|v\|_{q, T}^{q}\right\}^{\frac{1}{q}}\left\{h_{T}^{q}\|v\|_{q, T}^{q}\right\}^{\frac{1}{p}} \lesssim \sup _{w \in \mathbb{P}_{k}(T),\|w\|_{p, T}=\left\{h_{T}^{q}\|v\|_{q, T}^{q}\right\}^{\frac{1}{p}}}\left(v, h_{T} \Psi_{T} w\right)_{T} .
$$

Consequently, since the restrictions of functions in $\mathbb{P}_{k}\left(\mathcal{S}_{h}\right)$ to the elements of $\mathcal{S}_{h}$ can be chosen independently,

$$
\sum_{T \in \mathcal{S}_{h}} h_{T}^{q}\|v\|_{q, T}^{q} \lesssim \sup _{w \in \mathbb{P}_{k}\left(\mathcal{S}_{h}\right),\|w\|_{p, T}=\left\{h_{T}^{q}\|v\|_{q, T}^{q}\right\}^{\frac{1}{p}}} \sum_{\forall T \in \mathcal{S}_{h}} \sum_{T \in \mathcal{S}_{h}}\left(v, h_{T} \Psi_{T} w\right)_{T}
$$


whence (A.10) follows by extending the supremum set and rescaling the argument $w$. The following extension of (A.7c) is proven similarly: For all $\phi \in \mathbb{P}_{k}\left(\mathcal{G}_{h}^{\mathcal{T}}\right)$, the vector space spanned by piecewise polynomials of total degree $\leq k$ on each face of $\mathcal{G}_{h}^{\mathcal{T}}$,

$$
\left\{\sum_{F \in \mathcal{G}_{h}^{\mathcal{T}}} h_{F}\|\phi\|_{q, F}^{q}\right\}^{\frac{1}{q}} \lesssim \sup _{w \in \mathbb{P}_{k}\left(\mathcal{G}_{h}^{\mathcal{T}}\right),\|w\|_{p, \mathcal{G}_{h}^{\mathcal{T}}}=1} \sum_{F \in \mathcal{G}_{h}^{\mathcal{T}}}\left(\phi, h_{F}^{\frac{1}{q}} \Psi_{F} w\right)_{F},
$$

where $\|w\|_{p, \mathcal{G}_{h}^{\mathcal{T}}}:=\left\{\sum_{F \in \mathcal{G}_{h}^{\mathcal{T}}}\|w\|_{p, F}^{p}\right\}^{\frac{1}{p}}$.

We now prove that

$$
\left\{\sum_{T \in \mathcal{S}_{h}} h_{T}^{q}\left\|f+\nabla \cdot \boldsymbol{\sigma}_{L, h}\right\|_{q, T}^{q}\right\}^{\frac{1}{q}} \lesssim\left\|\nabla \cdot\left(\boldsymbol{\sigma}(\nabla u)-\boldsymbol{\sigma}_{L, h}\right)\right\|_{V^{\prime}} .
$$

Set $\left.v\right|_{T}:=\left.\left(f+\nabla \cdot \boldsymbol{\sigma}_{L, h}\right)\right|_{T}$ for all $T \in \mathcal{S}_{h}$, and observe that $v \in \mathbb{P}_{k}\left(\mathcal{S}_{h}\right)$. Let $w \in \mathbb{P}_{k}\left(\mathcal{S}_{h}\right)$ with $\|w\|_{p}=1$. Set $\left.\lambda\right|_{T}:=\left.h_{T} \Psi_{T} w\right|_{T}$ for all $T \in \mathcal{S}_{h}$, and observe that $\lambda \in V$ since $\Psi_{T}$ vanishes on $\partial T$, and that owing to (A.7b), $\|\lambda\|_{V}=\|\nabla \lambda\|_{p} \lesssim\|w\|_{p}=1$. Then, proceeding as before,

$$
\begin{aligned}
\sum_{T \in \mathcal{S}_{h}}\left(v, h_{T} \Psi_{T} w\right)_{T} & =\sum_{T \in \mathcal{S}_{h}}(v, \lambda)_{T}=\left(\boldsymbol{\sigma}(\nabla u)-\boldsymbol{\sigma}_{L, h}, \nabla \lambda\right) \\
& \leq\left\|\nabla \cdot\left(\boldsymbol{\sigma}(\nabla u)-\boldsymbol{\sigma}_{L, h}\right)\right\|_{V^{\prime}}\|\lambda\|_{V} \lesssim\left\|\nabla \cdot\left(\boldsymbol{\sigma}(\nabla u)-\boldsymbol{\sigma}_{L, h}\right)\right\|_{V^{\prime}},
\end{aligned}
$$

whence (A.12) follows from (A.10).

We next show that

$$
\left\{\sum_{F \in \mathcal{G}_{h}^{\mathcal{T}}} h_{F}\left\|\llbracket \boldsymbol{\sigma}_{L, h} \cdot \mathbf{n} \rrbracket\right\|_{q, F}^{q}\right\}^{\frac{1}{q}} \lesssim\left\|\nabla \cdot\left(\boldsymbol{\sigma}(\nabla u)-\boldsymbol{\sigma}_{L, h}\right)\right\|_{V^{\prime}} .
$$

Set $\left.\phi\right|_{F}:=\llbracket \boldsymbol{\sigma}_{L, h} \cdot \mathbf{n} \rrbracket_{F}$ for all $F \in \mathcal{G}_{h}^{\mathcal{T}}$ and observe that $\phi \in \mathbb{P}_{k}\left(\mathcal{G}_{h}^{\mathcal{T}}\right)$. Let $w \in \mathbb{P}_{k}\left(\mathcal{G}_{h}^{\mathcal{T}}\right)$ with $\|w\|_{p, \mathcal{G}_{h}^{\mathcal{T}}}=1$. Set $\left.\lambda\right|_{F}:=\left.h_{F}^{\frac{1}{q}} \Psi_{F} w\right|_{F}$ for all $F \in \mathcal{G}_{h}^{\mathcal{T}}$. This defines the function $\lambda$ on the set $\bigcup_{F \in \mathcal{G}_{h}^{\mathcal{T}}} \omega_{F}$ (recall that $\omega_{F} \subset \Omega$ denotes the union of the two simplices of $\mathcal{S}_{h}$ sharing $F$ ), and the function $\lambda$ is extended by zero outside this set. We first observe that $\lambda \in V$. Moreover, since for $F, F^{\prime} \in \mathcal{G}_{h}^{\mathcal{T}}, F \neq F^{\prime}, \omega_{F} \cap \omega_{F^{\prime}}$ has zero measure, it is inferred using (A.7e) that

$$
\left\{\sum_{T \in \mathcal{S}_{h}} h_{T}^{-p}\|\lambda\|_{p, T}^{p}\right\}^{\frac{1}{p}} \lesssim\left\{\sum_{F \in \mathcal{G}_{h}^{\tau}} h_{F}^{-p}\|\lambda\|_{p, \omega_{F}}^{p}\right\}^{\frac{1}{p}} \lesssim\|w\|_{p, \mathcal{G}_{h}^{T}}=1 .
$$

Similarly, owing to (A.7d), $\|\lambda\|_{V}=\|\nabla \lambda\|_{p} \lesssim 1$. As a result,

$$
\begin{aligned}
\sum_{F \in \mathcal{G}_{h}^{\mathcal{T}}}\left(\phi, h_{F}^{\frac{1}{q}} \Psi_{F} w\right)_{F} & =\sum_{F \in \mathcal{G}_{h}^{\mathcal{T}}}(\phi, \lambda)_{F}=\sum_{T \in \mathcal{S}_{h}}\left\{\left(f+\nabla \cdot \boldsymbol{\sigma}_{L, h}, \lambda\right)_{T}-\left(\boldsymbol{\sigma}(\nabla u)-\boldsymbol{\sigma}_{L, h}, \nabla \lambda\right)_{T}\right\} \\
& \leq\left\{\sum_{T \in \mathcal{S}_{h}} h_{T}^{q}\left\|f+\nabla \cdot \boldsymbol{\sigma}_{L, h}\right\|_{q, T}^{q}\right\}^{\frac{1}{q}}\left\{\sum_{T \in \mathcal{S}_{h}} h_{T}^{-p}\|\lambda\|_{p, T}^{p}\right\}^{\frac{1}{p}}+\left\|\nabla \cdot\left(\boldsymbol{\sigma}(\nabla u)-\boldsymbol{\sigma}_{L, h}\right)\right\|_{V^{\prime}}\|\lambda\|_{V} \\
& \lesssim\left\|\nabla \cdot\left(\boldsymbol{\sigma}(\nabla u)-\boldsymbol{\sigma}_{L, h}\right)\right\|_{V^{\prime}},
\end{aligned}
$$

owing to (A.12), whence (A.13) follows from (A.11).

Finally, (A.12) and (A.13) together with the triangle inequality imply that $\eta_{\text {res }} \lesssim\left\|\boldsymbol{\sigma}(\nabla u)-\boldsymbol{\sigma}_{L, h}\right\|_{V^{\prime}}$, and using a further triangle inequality leads to

$$
\left\|\nabla \cdot\left(\sigma(\nabla u)-\sigma_{L, h}\right)\right\|_{V^{\prime}} \leq\left\|\nabla \cdot\left(\sigma(\nabla u)-\sigma\left(\nabla u_{L, h}\right)\right)\right\|_{V^{\prime}}+\left\|\nabla \cdot\left(\sigma\left(\nabla u_{L, h}\right)-\sigma_{L, h}\right)\right\|_{V^{\prime}} .
$$


The first term in the right-hand side is equal to $\left\|A u-A u_{L, h}\right\|_{V^{\prime}}$, while using the Hölder inequality, the second term is bounded by $\eta_{\mathrm{L}}$ as defined by (3.10). This concludes the proof.

A.4. Proof of Lemma 5.3. Consider first the case of direct prescription. Let $D \in \mathcal{D}_{h}$ and let $T \in \mathcal{S}_{D}$ with outward unit normal $\mathbf{n}_{T}$. Then, for all $\mathbf{v}_{h} \in \mathbf{R T N}(T)$, the lowest-order Raviart-Thomas-Nédélec finite element space on $T$,

$$
\begin{aligned}
\left\|\mathbf{v}_{h}\right\|_{q, T} & \leq C_{d, 1, q}^{b}(T)|T|^{\frac{1}{q}-\frac{1}{2}}\left\|\mathbf{v}_{h}\right\|_{T} \leq C_{\kappa} h_{T}^{-\frac{1}{2}} C_{d, 1, q}^{b}(T)|T|^{\frac{1}{q}-\frac{1}{2}}\left\|\mathbf{v}_{h} \cdot \mathbf{n}_{T}\right\|_{\partial T} \\
& \leq C_{\kappa} C_{d, 1, q}^{b}(T) C_{d-1,0, q}^{\sharp}(\partial T) h_{T}^{-\frac{1}{2}}|T|^{\frac{1}{q}-\frac{1}{2}}|\partial T|^{\frac{1}{2}-\frac{1}{q}}\left\|\mathbf{v}_{h} \cdot \mathbf{n}_{T}\right\|_{q, \partial T} \\
& \leq C_{\kappa} C_{d, 1, q}^{b}(T) C_{d-1,0, q}^{\sharp}(\partial T)(|T| /|\partial T|)^{\frac{1}{q}}\left\|\mathbf{v}_{h} \cdot \mathbf{n}_{T}\right\|_{q, \partial T},
\end{aligned}
$$

where $|\partial T|$ denotes the $(d-1)$-dimensional Lebesgue measure of $\partial T$ and where the constant $C_{\kappa}$ results from the usual equivalence result in the $L^{2}$-setting for $\mathbf{R T N}(T)$ functions. Then, using the bounds (A.4)-(A.5) and since for all $k \geq 0, C_{d-1, k, \infty}^{\sharp}(\partial T) \leq C_{\kappa} C_{d-1, k}^{\star}$ where the constant $C_{d-1, k}^{\star}$ is defined as in (A.6) (indeed, for $v \in \mathbb{P}_{k}(\partial T)$, there is $F \subset \partial T$ such that $\|v\|_{\infty, \partial T}=\|v\|_{\infty, F}$ so that $\|v\|_{\infty, \partial T} \leq C_{d-1, k, \infty}^{\sharp}(F)|F|^{-1 / 2}\|v\|_{F} \leq C_{d-1, k, \infty}^{\sharp}(F) C_{\kappa}|\partial T|^{-1 / 2}\|v\|_{\partial T}$ and since $F$ is a simplex, $\left.C_{d-1, k, \infty}^{\sharp}(F) \leq C_{d-1, k}^{\star}\right)$, it is inferred that

$$
\left\|\mathbf{v}_{h}\right\|_{q, T} \leq C_{\kappa, d}(|T| /|\partial T|)^{\frac{1}{q}}\left\|\mathbf{v}_{h} \cdot \mathbf{n}_{T}\right\|_{q, \partial T}
$$

where $C_{\kappa, d}$ denotes a generic constant only depending on $\kappa$ and $d$. Finally, since $|T| \leq C_{\kappa} h_{T}|\partial T|$ and since $\frac{1}{q} \leq 1$, this yields

$$
\left\|\mathbf{v}_{h}\right\|_{q, T} \leq C_{\kappa, d} h_{T}^{\frac{1}{q}}\left\|\mathbf{v}_{h} \cdot \mathbf{n}_{T}\right\|_{q, \partial T}
$$

We now apply this estimate to $\mathbf{v}_{h}:=\sigma_{L, h}+\mathbf{t}_{h}$. Then, owing to (5.3)-(5.4),

$$
\left\|\boldsymbol{\sigma}_{L, h}+\mathbf{t}_{h}\right\|_{q, T}^{q} \lesssim h_{T} \sum_{F \subset \partial T, F \in \mathcal{G}_{D}^{\mathcal{T}}}\left\|\llbracket \boldsymbol{\sigma}_{L, h} \cdot \mathbf{n} \rrbracket\right\|_{q, F}^{q} .
$$

Hence, $\eta_{\mathrm{DF}, D}^{q} \lesssim \sum_{F \in \mathcal{G}_{D}^{\mathcal{T}}} h_{F}\left\|\llbracket \boldsymbol{\sigma}_{L, h} \cdot \mathbf{n} \rrbracket\right\|_{q, F}^{q}$, whence (4.2) follows.

We now consider the case of local linear system solves. Let $D \in \mathcal{D}_{h}$. Using the approach of [39, Section 4.1] (cf. also [3, 2]), there exists a postprocessing $\tilde{q}_{h} \in M\left(\mathcal{S}_{D}\right)$ of $q_{h}$ such that

$$
\begin{array}{rlrl}
-\nabla \tilde{q}_{h} & =\boldsymbol{\sigma}_{L, h}+\mathbf{t}_{h} & & \forall T \in \mathcal{S}_{D}, \\
\frac{\left(\tilde{q}_{h}, 1\right)_{T}}{|T|} & =\left.q_{h}\right|_{T} & \forall T \in \mathcal{S}_{D} .
\end{array}
$$

Here, $M\left(\mathcal{S}_{D}\right)$ is a space of particular piecewise polynomials on $\mathcal{S}_{D}$ of total degree $\leq 2$ whose means of traces on interior faces are continuous and whose mean value over $D$ is zero when $D \in \mathcal{D}_{h}^{\text {int }}$ and whose mean values over faces lying in $\partial \Omega$ are zero when $D \in \mathcal{D}_{h}^{\text {ext }}$. Then,

$$
\left\|\boldsymbol{\sigma}_{L, h}+\mathbf{t}_{h}\right\|_{q, D} \lesssim \sup _{m_{h} \in M\left(\mathcal{S}_{D}\right),\left\|\nabla m_{h}\right\|_{p, D}=1}\left(\boldsymbol{\sigma}_{L, h}+\mathbf{t}_{h}, \nabla m_{h}\right)_{D}
$$

Indeed, $\left\|\boldsymbol{\sigma}_{L, h}+\mathbf{t}_{h}\right\|_{q, D}=\left(\nabla \tilde{q}_{h}, \nabla \tilde{m}_{h}\right)_{D}$ with $\tilde{m}_{h}=\left(\left\|\nabla \tilde{q}_{h}\right\|_{q, D} /\left\|\nabla \tilde{q}_{h}\right\|_{D}^{2}\right) \tilde{q}_{h}$, so that introducing constants as in (A.2) yields

$$
\left\|\nabla \tilde{m}_{h}\right\|_{p, D}=\frac{\left\|\nabla \tilde{q}_{h}\right\|_{q, D}\left\|\nabla \tilde{q}_{h}\right\|_{p, D}}{\left\|\nabla \tilde{q}_{h}\right\|_{D}^{2}} \leq C_{d, 2, p}^{\sharp}(D) C_{d, 2, q}^{\sharp}(D),
$$


and bounding the constants by $C_{d, 2, \infty}(D)$ and using the shape-regularity of the submesh of the dual volume $D$ leads, as before, to the bound $\left\|\nabla \tilde{m}_{h}\right\|_{p, D} \lesssim 1$. We now develop the right-hand side of (A.14),

$$
\begin{aligned}
\left(\boldsymbol{\sigma}_{L, h}+\mathbf{t}_{h}, \nabla m_{h}\right)_{D}= & \sum_{T \in \mathcal{S}_{D}}\left\{-\left(m_{h}, \nabla \cdot\left(\boldsymbol{\sigma}_{L, h}+\mathbf{t}_{h}\right)\right)_{T}+\left(\left(\boldsymbol{\sigma}_{L, h}+\mathbf{t}_{h}\right) \cdot \mathbf{n}, m_{h}\right)_{\partial T}\right\} \\
= & -\sum_{T \in \mathcal{S}_{D}}\left(m_{h}, f+\nabla \cdot \boldsymbol{\sigma}_{L, h}\right)_{T}+\sum_{F \in \mathcal{G}_{D}^{\mathcal{T}}}\left(\llbracket \boldsymbol{\sigma}_{L, h} \cdot \mathbf{n} \rrbracket, m_{h}\right)_{F} \\
\leq & \left\{\sum_{T \in \mathcal{S}_{D}} h_{T}^{-p}\left\|m_{h}\right\|_{p, T}^{p}\right\}^{\frac{1}{p}}\left\{\sum_{T \in \mathcal{S}_{D}} h_{T}^{q}\left\|f+\nabla \cdot \boldsymbol{\sigma}_{L, h}\right\|_{q, T}^{q}\right\}^{\frac{1}{q}} \\
& +\left\{\sum_{F \in \mathcal{G}_{D}^{\mathcal{T}}} h_{F}^{-\frac{p}{q}}\left\|m_{h}\right\|_{p, F}^{p}\right\}^{\frac{1}{p}}\left\{\sum_{F \in \mathcal{G}_{D}^{\mathcal{T}}} h_{F}\left\|\llbracket \boldsymbol{\sigma}_{L, h} \cdot \mathbf{n} \rrbracket\right\|_{q, F}^{q}\right\}^{\frac{1}{q}} \\
\lesssim & h_{D}^{-1}\left\|m_{h}\right\|_{p, D}\left\{\sum_{T \in \mathcal{S}_{D}} h_{T}^{q}\left\|f+\nabla \cdot \boldsymbol{\sigma}_{L, h}\right\|_{q, T}^{q}+\sum_{F \in \mathcal{G}_{D}^{\mathcal{T}}} h_{F}\left\|\llbracket \boldsymbol{\sigma}_{L, h} \cdot \mathbf{n} \rrbracket\right\| \|_{q, F}^{q}\right\}^{\frac{1}{q}},
\end{aligned}
$$

using the Green theorem, the fact that $\nabla \cdot \mathbf{t}_{h}=f$ for all $T \in \mathcal{S}_{D}$ owing to (5.6b), the fact that $\left.\llbracket \mathbf{t}_{h} \cdot \mathbf{n} \rrbracket\right|_{F}=0$ for all $F \in \mathcal{G}_{D}^{\mathcal{T}}$ since $\mathbf{t}_{h} \in \mathbf{R T N}_{\mathrm{N}}\left(\mathcal{S}_{D}\right)$, the Hölder inequality, and the inverse inequality

$$
\left\|m_{h}\right\|_{p, F} \lesssim h_{F}^{-\frac{1}{p}}\left\|m_{h}\right\|_{p, T}
$$

which can be proven by proceeding as above using the usual inverse inequality in the $L^{2}$-setting and the norm equivalence constants. We now use the discrete Poincaré/Friedrichs inequality (recall that $\left(m_{h}, 1\right)_{D}=0$ or that $\left(m_{h}, 1\right)_{\partial D \cap \partial \Omega}=0$ since $\left.m_{h} \in M\left(\mathcal{S}_{D}\right)\right)$

$$
\left\|m_{h}\right\|_{p, D} \lesssim h_{D}\left\|\nabla m_{h}\right\|_{p, D}
$$

which can be proven by proceeding as above using the usual discrete Poincaré/Friedrichs inequality in the $L^{2}$-setting (for nonconvex $D$, an upper bound only depending on $\kappa$ can be inferred from [38]) and the norm equivalence constants. Then,

$$
\left(\boldsymbol{\sigma}_{L, h}+\mathbf{t}_{h}, \nabla m_{h}\right)_{D} \lesssim\left\{\sum_{T \in \mathcal{S}_{D}} h_{T}^{q}\left\|f+\nabla \cdot \boldsymbol{\sigma}_{L, h}\right\|_{q, T}^{q}+\sum_{F \in \mathcal{G}_{D}^{\mathcal{T}}} h_{F}\left\|\llbracket \boldsymbol{\sigma}_{L, h} \cdot \mathbf{n} \rrbracket\right\|_{q, F}^{q}\right\}^{\frac{1}{q}},
$$

and (4.2) now follows from (A.14).

\section{REFERENCES}

[1] Ainsworth, M., And Oden, J. T. A posteriori error estimation in finite element analysis. Pure and Applied Mathematics (New York). Wiley-Interscience [John Wiley \& Sons], New York, 2000.

[2] Arbogast, T., And Chen, Z. On the implementation of mixed methods as nonconforming methods for second-order elliptic problems. Math. Comp. 64, 211 (1995), 943-972.

[3] Arnold, D. N., And Brezzi, F. Mixed and nonconforming finite element methods: implementation, postprocessing and error estimates. RAIRO Modél. Math. Anal. Numér. 19, 1 (1985), 7-32.

[4] Bank, R. E., And Rose, D. J. Some error estimates for the box method. SIAM J. Numer. Anal. 24, 4 (1987), $777-787$.

[5] Bank, R. E., And Weiser, A. Some a posteriori error estimators for elliptic partial differential equations. Math. Comp. 44, 170 (1985), 283-301.

[6] Barrett, J. W., And LiU, W. B. Finite element approximation of the p-Laplacian. Math. Comp. 61, 204 (1993), $523-537$.

[7] Braess, D., And Schöberl, J. Equilibrated residual error estimator for edge elements. Math. Comp. 77, 262 (2008), $651-672$.

[8] Brezzi, F., And Fortin, M. Mixed and hybrid finite element methods, vol. 15 of Springer Series in Computational Mathematics. Springer-Verlag, New York, 1991. 
[9] Carstensen, C., And Klose, R. A posteriori finite element error control for the p-Laplace problem. SIAM J. Sci. Comput. 25, 3 (2003), 792-814 (electronic).

[10] Carstensen, C., Liu, W., and Yan, N. A posteriori FE error control for $p$-Laplacian by gradient recovery in quasinorm. Math. Comp. 75, 256 (2006), 1599-1616 (electronic).

[11] Challlou, A., And Suri, M. A posteriori estimation of the linearization error for strongly monotone nonlinear operators. J. Comput. Appl. Math. 205, 1 (2007), 72-87.

[12] Chaillou, A. L., AND Suri, M. Computable error estimators for the approximation of nonlinear problems by linearized models. Comput. Methods Appl. Mech. Engrg. 196, 1-3 (2006), 210-224.

[13] Ciarlet, P. G. The finite element method for elliptic problems, vol. 40 of Classics in Applied Mathematics. Society for Industrial and Applied Mathematics (SIAM), Philadelphia, PA, 2002. Reprint of the 1978 original [North-Holland, Amsterdam; MR0520174 (58 \#25001)].

[14] Destuynder, P., And MÉtivet, B. Explicit error bounds in a conforming finite element method. Math. Comp. 68, 228 (1999), 1379-1396.

[15] Diening, L., And EtTwein, F. Fractional estimates for non-differentiable elliptic systems with general growth. Forum Math. 20, 3 (2008), 523-556.

[16] Diening, L., And Kreuzer, C. Linear convergence of an adaptive finite element method for the $p$-Laplacian equation. SIAM J. Numer. Anal. 46, 2 (2008), 614-638.

[17] Ern, A., And Guermond, J.-L. Theory and Practice of Finite Elements, vol. 159 of Applied Mathematical Sciences. Springer-Verlag, New York, NY, 2004.

[18] ERn, A., And Vohralík, M. Flux reconstruction and a posteriori error estimation for discontinuous Galerkin methods on general nonmatching grids. C. R. Math. Acad. Sci. Paris 347 (2009), 441-444.

[19] Glowinski, R., and Marrocco, A. Sur l'approximation, par éléments finis d'ordre 1, et la résolution, par pénalisationdualité, d'une classe de problèmes de Dirichlet non linéaires. C. R. Acad. Sci. Paris Sér. A 278 (1974), 1649-1652.

[20] Glowinski, R., And Marrocco, A. Sur l'approximation, par éléments finis d'ordre un, et la résolution, par pénalisation-dualité, d'une classe de problèmes de Dirichlet non linéaires. Rev. Française Automat. Informat. Recherche Opérationnelle (RAIRO Analyse Numérique) 9, R-2 (1975), 41-76.

[21] Jiránek, P., Strakoš, Z., And Vohralík, M. A posteriori error estimates including algebraic error: computable upper bounds and stopping criteria for iterative solvers. Preprint R08045, Laboratoire Jacques-Louis Lions \& HAL Preprint 00326650, submitted for publication, 2008.

[22] KIM, K. Y. A posteriori error estimators for locally conservative methods of nonlinear elliptic problems. Appl. Numer. Math. 57 (2007), 1065-1080.

[23] Ladevèze, P., And Leguillon, D. Error estimate procedure in the finite element method and applications. SIAM J. Numer. Anal. 20, 3 (1983), 485-509.

[24] LIU, W., AND YAN, N. Quasi-norm a priori and a posteriori error estimates for the nonconforming approximation of p-Laplacian. Numer. Math. 89, 2 (2001), 341-378.

[25] LiU, W., AND YAN, N. Quasi-norm local error estimators for p-Laplacian. SIAM J. Numer. Anal. 39, 1 (2001), $100-127$.

[26] LIU, W., AND YAN, N. On quasi-norm interpolation error estimation and a posteriori error estimates for $p$-Laplacian. SIAM J. Numer. Anal. 40, 5 (2002), 1870-1895.

[27] Luce, R., And Wohlmuth, B. I. A local a posteriori error estimator based on equilibrated fluxes. SIAM J. Numer. Anal. 42, 4 (2004), 1394-1414.

[28] Veeser, A. Convergent adaptive finite elements for the nonlinear Laplacian. Numer. Math. 92, 4 (2002), 743-770.

[29] Verfürth, R. A posteriori error estimates for nonlinear problems. Finite element discretizations of elliptic equations. Math. Comp. 62, 206 (1994), 445-475.

[30] Verfürth, R. A review of a posteriori error estimation and adaptive mesh-refinement techniques. Teubner-Wiley, Stuttgart, 1996.

[31] Verfürth, R. A posteriori error estimates for nonlinear problems. $L^{r}$-estimates for finite element discretizations of elliptic equations. RAIRO Modél. Math. Anal. Numér. 32, 7 (1998), 817-842.

[32] Verfürth, R. A note on polynomial approximation in Sobolev spaces. M2AN Math. Model. Numer. Anal. 33, 4 (1999), $715-719$.

[33] Verfürth, R. On the constants in some inverse inequalities for finite element functions. Tech. report, Ruhr-Universität Bochum, 1999.

[34] Verfürth, R. A posteriori error estimates for finite element discretizations of the heat equation. Calcolo 40, 3 (2003), $195-212$.

[35] Verfürth, R. A posteriori error estimates for non-linear parabolic equations. Tech. report, Ruhr-Universität Bochum, 2004.

[36] Verfürth, R. Robust a posteriori error estimates for stationary convection-diffusion equations. SIAM J. Numer. Anal. 43, 4 (2005), 1766-1782.

[37] VERFüRTh, R. personnal communication. 2009. 
[38] Vohralík, M. On the discrete Poincaré-Friedrichs inequalities for nonconforming approximations of the Sobolev space $H^{1}$. Numer. Funct. Anal. Optim. 26, 7-8 (2005), 925-952.

[39] Vohralík, M. A posteriori error estimates for lowest-order mixed finite element discretizations of convection-diffusionreaction equations. SIAM J. Numer. Anal. 45, 4 (2007), 1570-1599.

[40] Vohralík, M. Guaranteed and fully robust a posteriori error estimates for conforming discretizations of diffusion problems with discontinuous coefficients. Preprint R08009, Laboratoire Jacques-Louis Lions \& HAL Preprint 00235810, submitted for publication, 2008. 